Published in final edited form as:

Cancer Discov. 2016 January ; 6(1): 80-95. doi:10.1158/2159-8290.CD-15-0224.

\title{
Targeting YAP-dependent MDSC infiltration impairs tumor progression
}

\author{
Guocan Wang ${ }^{1,2,{ }^{*}}$, Xin $\mathbf{L u}^{1,2,{ }^{*}, \text { Prasenjit Dey }}{ }^{1,2}$, Pingna Deng ${ }^{1,2}$, Chia Chin $\mathbf{W u}^{3}$, Shan \\ Jiang $^{2}$, Zhuangna Fang ${ }^{2,7}$, Kun Zhao ${ }^{2}$, Ramakrishna Konaparthi ${ }^{2}$, Sujun Hua ${ }^{1,2}$, Jianhua \\ Zhang $^{3}$, Elsa M. Li-Ning-Tapia ${ }^{4}$, Avnish Kapoor ${ }^{2}$, Chang-Jiun $\mathrm{Wu}^{3}$, Neelay Bhaskar Patel ${ }^{2}$, \\ Zhenglin Guo ${ }^{1}$, Vandhana Ramamoorthy ${ }^{3}$, Trang N. Tieu ${ }^{3}$, Tim Heffernan ${ }^{3}$, Di Zhao ${ }^{1,2}$, \\ Xiaoying Shang ${ }^{1}$, Sunada Khadka ${ }^{1}$, Pingping Hou ${ }^{1,2}$, Baoli Hu${ }^{1,2}$, Eun-Jung Jin ${ }^{1,8}$, Wantong \\ $\mathrm{Yao}^{2}$, Xiaolu Pan ${ }^{2}$, Zhihu Ding ${ }^{9}$, Yanxia Shi ${ }^{2,7}$, Liren $\mathrm{Li}^{2,7}$, Qing Chang ${ }^{3}$, Patricia Troncoso ${ }^{5}$, \\ Christopher J. Logothetis ${ }^{4}$, Mark J. McArthur ${ }^{6}$, Lynda Chin ${ }^{2}$, Y. Alan Wang ${ }^{1,2}$, and Ronald \\ A. DePinho ${ }^{1}$ \\ ${ }^{1}$ Department of Cancer Biology, University of Texas MD Anderson Cancer Center, 1515 \\ Holcombe Boulevard, Houston, TX 77030, USA \\ 2Department of Genomic Medicine, University of Texas MD Anderson Cancer Center, 1515 \\ Holcombe Boulevard, Houston, TX 77030, USA \\ ${ }^{3}$ Institute for Applied Cancer Science, University of Texas MD Anderson Cancer Center, 1515 \\ Holcombe Boulevard, Houston, TX 77030, USA \\ ${ }^{4}$ Department of Genitourinary Medical Oncology, University of Texas MD Anderson Cancer \\ Center, 1515 Holcombe Boulevard, Houston, TX 77030, USA \\ ${ }^{5}$ Department of Pathology, University of Texas MD Anderson Cancer Center, 1515 Holcombe \\ Boulevard, Houston, TX 77030, USA \\ ${ }^{6}$ Department of Veterinary Medicine \& Surgery, University of Texas MD Anderson Cancer Center, \\ 1515 Holcombe Boulevard, Houston, TX 77030, USA
}

Correspondence should be addressed to: Ronald A. DePinho and Y. Alan Wang, Department of Cancer Biology, University of Texas MD Anderson Cancer Center, 1515 Holcombe Boulevard, Houston, TX 77030, USA. Y.A. W: yalanwang @ mdanderson.org/ (713) 792-7928; R.A. D: rdepinho@mdanderson.org/(713) 792-6000.

These authors contributed equally to this work

Conflict of Interest: The authors disclose no potential conflicts of interest.

Accession Numbers: The expression array data used in this manuscript was in GEO with accession number GSE25140 (11) and GSE71319.

Author Contributions: Conception and design: G. Wang, X. Lu, P. Dey, Y.A. Wang and R.A. DePinho. Development of methodology: G. Wang, X. Lu, P. Dey.

Acquisition of data (provided animals, acquired and managed patients, provided facilities, etc.): G. Wang, X. Lu, P. Dey, E. Tapia, M.J. McArthur, P. Deng, R. Konaparthi, S. Hua, N.B. Patel, Z. Guo, P. Hou, D. Zhao, X. Shang, S. Khadka, B. Hu, Q. Chang, X. Pan, Z. Ding, Y. Shi, L. Li, M. J. McArthur, E. Tapia, P. Troncoso.

Analysis and interpretation of data (e.g., statistical analysis, biostatistics, computational analysis): G. Wang, X. Lu, P. Dey, C.C.

Wu, C.J. Wu, Y.A. Wang and R.A. DePinho.

Writing, review, and/or revision of the manuscript: G. Wang, X. Lu, P. Dey, Y.A. Wang and R.A. DePinho.

Administrative, technical, or material support (i.e., reporting or organizing data, constructing databases): S. Jiang, Z. Fang, K.

Zhao, E. Jin, W. Yao, A. Kapoor, T. N. Tieu, V. Ramamoorthy, T. Heffernan, C.C. Wu, C.J. Wu, L. Chin.

Study supervision: G. Wang, X. Lu, J. Zhang, C.J. Logothetis, Y.A. Wang, R.A. DePinho.

Other (performed pathology): M. J. McArthur, E. Tapia, P. Troncoso. 
${ }^{7}$ Sun Yat-Sen University Cancer Center, Guangzhou, People's Republic of China

${ }^{8}$ Department of Biological Science, College of Natural Sciences, Wonkwang University, Cheonbuk, Iksan, South Korea, 570-749

${ }^{9}$ Sanofi Oncology, 640 Memorial Drive, Cambridge, MA, 02139, USA

\section{Abstract}

The signaling mechanisms between prostate cancer cells and infiltrating immune cells may illuminate novel therapeutic approaches. Here, utilizing a prostate adenocarcinoma model driven by loss of Pten and Smad4, we identify polymorphonuclear myeloid-derived suppressor cells (MDSCs) as the major infiltrating immune cell type and depletion of MDSCs blocks progression. Employing a novel dual reporter prostate cancer model, epithelial and stromal transcriptomic profiling identified Cxc15 as a cancer-secreted chemokine to attract Cxcr2-expressing MDSCs and, correspondingly, pharmacological inhibition of Cxcr2 impeded tumor progression. Integrated analyses identified hyperactivated Hippo-YAP signaling in driving Cxc15 upregulation in cancer cells through YAP-TEAD complex and promoting MDSCs recruitment. Clinico-pathological studies reveal upregulation and activation of YAP1 in a subset of human prostate tumors, and the YAP1 signature is enriched in primary prostate tumor samples with stronger expression of MDSC relevant genes. Together, YAP-driven MDSC recruitment via heterotypic Cxc15-Cxcr2 signaling reveals effective therapeutic strategy for advanced prostate cancer.

Significance-We demonstrate a critical role of MDSCs in prostate tumor progression and discover a cancer cell non-autonomous function of Hippo-YAP pathway in regulation of $\mathrm{Cxcl5}$, a ligand for Cxcr2 expressing MDSCs. Pharmacologic elimination of MDSCs or blocking the heterotypic $\mathrm{CxCl5}-\mathrm{Cxcr} 2$ signaling circuit elicits robust anti-tumor responses and prolongs survival.

\section{Keywords}

Cancer; prostate; genetics Yap1; MDSCs; Cxcr2; Pten; Smad4

\section{Introduction}

The tumor microenvironment (TME) is comprised of a complex mixture of tumor-associated fibroblasts, infiltrating immune cells, endothelial cells and extracellular matrix proteins and signaling molecules such as cytokines (1-3). Homotypic and heterotypic interactions between these cellular constituents play essential roles in cancer development and response to therapeutics $(3,4)$. Among the infiltrating immune cells, MDSCs represent a phenotypically heterogeneous population of immature myeloid cells that play a tumorpromoting role by maintaining a state of immunological anergy and tolerance (5). In particular, activated MDSCs provide a source of secreted chemokines, cytokines and enzymes which suppress local T cell activation and viability (5). In addition, MDSCs can suppress $\mathrm{T}$ cell activity through deprivation of nutrients, such as L-arginine and L-cysteine, and interference with $\mathrm{T}$ cell receptor functions via reactive oxygen species (ROS) and reactive nitrogen species. 
Prostate cancer ( $\mathrm{PCa})$ is the most common noncutaneous malignancy in men in the United States. Similar to many other solid tumor types, $\mathrm{PCa}$ is characterized by a rich tumor-stroma interaction network that forms the TME (1-3). In PCa, various signaling pathways have been implicated in the crosstalk between tumor and stroma, such as androgen receptor signaling, FGF, Src, TGF $\beta$, IGF, integrin and Hedgehog pathways (1). Interestingly, MDSC abundance in the blood correlates with circulating Prostate Specific Antigen (PSA) levels in PCa patients (6-8). MDSCs have been identified recently as a TME constituent in an indolent prostate cancer mouse model with conditional Pten deletion (9) and demonstrated to antagonize senescence during early tumorigenesis (10). However, the molecular mechanisms underlying the recruitment of MDSCs are not well understood and the extent to which MDSCs facilitate PCa progression has not been determined.

Previously, we have shown that deletion of Pten in the mouse prostate causes upregulation of Smad4 which constrains cell proliferation and invasion and, accordingly, dual deletion of Pten and Smad4 results in rapid PCa progression including metastasis (11). Comparative transcriptomic and cell profile analyses of Pten versus Pten/Smad4 deficient PCa revealed a prominent immune signature and resident MDSCs as a major TME population in Pten/ Smad4 deficient tumors. Biological, molecular and pharmacological analyses established that a Yap1-mediated Cxc15-Cxcr2 signaling axis recruits MDSCs into the TME and that MDSCs play critical roles in facilitating tumor progression. Our comprehensive analyses using PCa model coupled with clinical validation using patients samples support the view that targeting either MDSC recruitment or infiltrated MDSCs may represent a valid therapeutic opportunity in treating advanced prostate cancer.

\section{Results}

\section{Prominent Infiltration of Immune Cells in the Pten ${ }^{p c-/-S m a d 4^{p c-/-}}$ Tumor Model}

We previously reported that conditional deletion of Smad4 bypassed the senescence barrier instigated by Pten loss in the prostate epithelia, resulting in a highly proliferative and invasive prostate adenocarcinoma characterized by an exuberant stromal reaction and frequent metastasis to distant organs (11). Correspondingly, Ingenuity Pathway Analysis (IPA) revealed prominent representation of cell movement, cell proliferation, and antigen

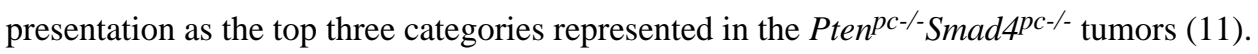
Further analysis revealed a prominent immune signature including Granulocytes Adhesion and Diapedesis, Leukocytes Extravasation Signaling, and Agrandulocytes Adhesion and

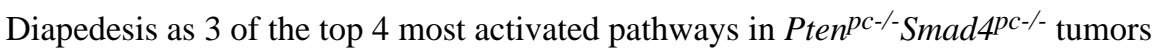
compared to those present in Pten ${ }^{\text {pc-/ }}$ tumors (Figure 1A; p value $<2.03 \mathrm{E}-7$ ).

Correspondingly, immunohistochemical staining (IHC) highlighted conspicuous infiltration of CD45 ${ }^{+}$leukocytes in Pten ${ }^{p c-/-S m a d 4 p c-/-~ t u m o r s ~(F i g u r e ~ 1 B) . ~ T o ~ c o m p r e h e n s i v e l y ~ a u d i t ~}$ the spectrum of infiltrating immune cells in tumors, we performed mass cytometry (CyTOF) immunophenotyping (12) to catalog tumor cell type constituents from well-established

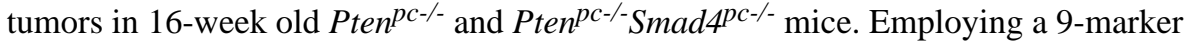
antibody panel (Supplementary Table S1), CyTOF confirmed a significant increase of CD45 ${ }^{+}$infiltrating leukocytes in Pten ${ }^{\text {pc- }-}$ Smad $4^{p c-/}$ as compared to Pten ${ }^{\text {pc- }}$ - tumors (Figure 1C). Within the $\mathrm{CD} 45^{+}$infiltrating cells, $\mathrm{CD} 11 \mathrm{~b}^{+}$myeloid cells represented a significantly 


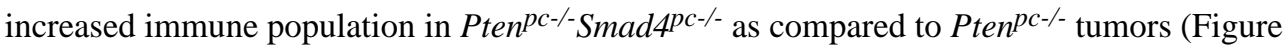
1D).

\section{CD $11 b^{+}$Gr-1 ${ }^{+}$Cells are Significantly Increased in Pten ${ }^{p c-/-S m a d 4 p c-/-~ T u m o r ~ M o d e l ~}$}

To obtain a dynamic view of peripheral and infiltrating immune cells as a function of tumor progression in the Pten ${ }^{p c-/-S m a d 4 p c-/-}$ model, which initiates tumor development at 6-8 weeks and progresses to early invasive carcinoma by 14 weeks of age, serial CyTOF analyses using an expanded antibody panel of 17 surface markers (Supplementary Table S1) were performed on single cells from primary tumors, peripheral blood, spleen and draining lymph nodes at 5,8 , and 14 weeks of age. The detailed immunophenotyping profiles enabled construction of the SPADE derived tree (12). SPADE (spanning-tree progression analysis of density-normalized events) is a computational approach to facilitate the

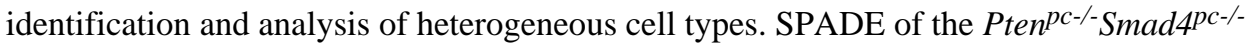
model displays the complexity of the TME which is composed of epithelial tumor cells $\left(\mathrm{EpCAM}^{+} \mathrm{CD}^{-} 5^{-}\right)$, non-immune TME cells (EpCAM- $\mathrm{CD}^{-}$), and infiltrating immune cells $\left(\mathrm{EpCAM}^{-} \mathrm{CD}^{+} 5^{+}\right)$that can be further grouped into various immune cell subpopulations (Figure 2A and Supplementary Figure S1A). Among the infiltrating immune cells, there was a striking age-dependent increase of $\mathrm{CD} 11 \mathrm{~b}^{+} \mathrm{Gr}-1^{+}$cells in tumors (Figure 2B) and

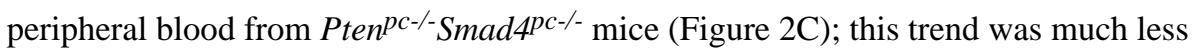
pronounced in the spleen or draining lymph nodes (Supplementary Figure S1B, for gating strategy see Supplementary Figure S1C).

\section{CD11b+ Gr-1+ Cells from Pten ${ }^{p c-/-S m a d 4 p c-/-~ t u m o r s ~ a r e ~ p o t e n t l y ~ i m m u n o s u p p r e s s i v e ~}$}

To evaluate the potential immunosuppressive activity of intratumoral CD $11 \mathrm{~b}^{+} \mathrm{Gr}-1^{+}$cells

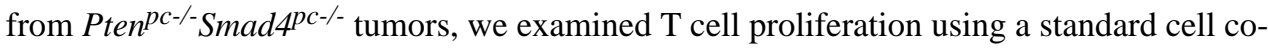
culture system. These CD11 b ${ }^{+}$Gr- $1^{+}$cells strongly suppressed CD3 and CD28 antibodyinduced T cell proliferation and activation (Figure 3A-B, see Supplementary Figure S2 for cell isolation strategy), establishing that $\mathrm{CD} 11 \mathrm{~b}^{+} \mathrm{Gr}-1^{+}$cells are indeed functional MDSCs.

MDSCs can be further classified as Ly- $6 \mathrm{G}^{+} \mathrm{Ly}-6 \mathrm{C}^{\mathrm{Low}}$ subset with polymorphonuclear phenotype (PMN-MDSCs) and Ly-6G- Ly-6 $\mathrm{C}^{\text {high }}$ subset with monocytic phenotype (M-

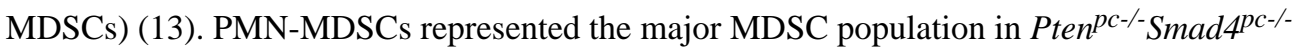
tumors (Figure 3C-D), consistent with previously observed preferential expansion of PMNMDSCs in tumor-bearing mice of various syngeneic models $(5,9,13)$. The abundance of PMN-MDSCs was further confirmed by IHC for Ly-6G, as shown by quantification of both

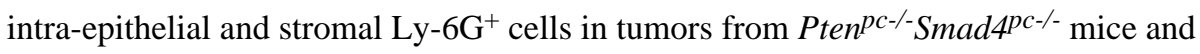
Pten $^{\text {pc-/ }}$ mice (Figure 3E-F). It has been shown previously that ROS production by PMNMDSCs is one of the mechanisms driving immune suppression (5, 14-16). Correspondingly, IPA revealed that pathways involved in ROS and NO production are among the top

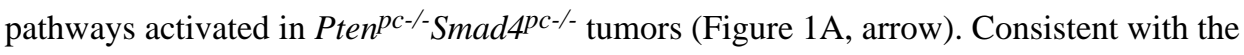

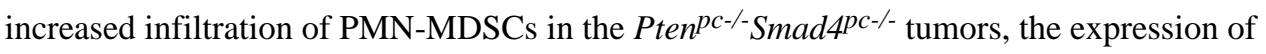
several subunits of NADPH oxidase (Nox2, p40 phox and p47phox), which are responsible for

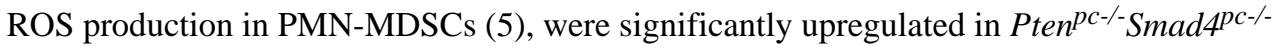
tumors relative to Pten $^{p c-/-}$ tumors (Figure 3G). Moreover, Arg1, but not Nos2, was highly upregulated in the Pten ${ }^{p c-/}$ Smad4 $4^{\text {pc- }}$ tumors (Figure 3G). Together, MDSCs in 
autochthonous $\mathrm{Pten}^{\text {pc-/-Smad4pc-/ }}$ tumors display strong T cell suppressive activity and are predominantly the PMN-MDSC subtype.

\section{Immuno-depletion of MDSCs Impedes Tumor Progression in Pten ${ }^{p c-/-S m a d 4^{p c-/-} \text { Mice }}$}

Enrichment of MDSCs in advanced Pten ${ }^{\text {pc-/ }}$ Smad4pc-/- tumors prompted us to explore the possible role of MDSCs in tumor progression. Using a well-characterized anti-Gr-1 neutralizing monoclonal antibody (clone RB6-8C5) (17), MDSCs were depleted in $\mathrm{Pten}^{\text {pc-/-Smad4 }}{ }^{\text {pc-/ }}$ mice at 14 weeks of age, a point coincident with progression to the early invasive carcinoma stage (see Supplementary Figure S3A for treatment scheme). The potent MDSC depletion activity of anti-Gr-1 monoclonal antibody was evidenced by significantly decreased PMN-MDSCs and M-MDSCs in peripheral blood as early as day 2 post-treatment (Supplementary Figure S3B). Additionally, a systemic reduction of MDSCs in spleen, bone marrow, and prostate tumors was documented following a 30-day treatment regimen of antiGr-1 monoclonal antibody (Figure 4A and Supplementary Figure S3C). This MDSC depletion was accompanied by an increase of $\mathrm{CD}^{+} \mathrm{T}$ cells (so-called killer $\mathrm{T}$ cells) (Figure 4A), consistent with elimination of the T cell suppression activity of MDSCs. Importantly, in line with the $\mathrm{CD}^{+} \mathrm{T}$ cell expansion, we observed that the $\mathrm{Gr}-1$ treated prostate displayed remarkable weight reduction in ventral and dorsolateral prostate (VP \& DLP) (Figure 4B).

The lack of difference in the weight of anterior prostate (AP) is likely due to the fact that AP tend to developed cysts with fluid accumulated inside the gland $(18,19)$, which also prevent the accurate measure of the prostate weight (Supplementary Figure S3D). Histopathological analysis revealed adenocarcinoma was the predominant pathology in mice treated with the control IgG whereas mPIN was the predominant morphological presentation in prostates from mice treated with anti-Gr-1 monoclonal antibody (Figure 4C \& Supplementary Table S2). In addition, by immunohistochemical staining for CD45, Ki67, vimentin, smooth muscle actin (SMA), and Trichrome staining, we observed that tumor remnants in mice treated with anti-Gr-1 monoclonal antibody displayed markedly reduced levels of cellular proliferation, stromal reaction, and inflammation as compared to those tumors treated with control IgG antibody (Supplementary Figure S4A).

In another therapeutic trial, we also utilized the recently developed MDSC-specific peptideFc fusion protein (i.e., peptibodies) that have been shown to effectively eliminate MDSCs in vivo through targeting the S100A9 surface protein (20). Employing a hydrodynamic injection approach for nucleic acid delivery (21), intravenous injection of either Pep-H6 peptibody expression vector or irrelevant control peptibody vector were initiated at 14 weeks every 4 days in Pten ${ }^{p c-/}$ Smad $4^{p c-/}$ mice. Strikingly, a single injection of the Pep-H6 peptibody significantly reduced the MDSCs in the peripheral blood, whereas such effect was not observed using the irrelevant control peptibody (Supplementary Figure S4B). Pep-H6 peptibody treatment for one month led to a dramatic decrease in cancer cell content in the prostate tumors (Figure 4D) and provided significant survival benefit for tumor-bearing mice (Figure 4E). Together, our data strongly support the view that MDSC depletion blocks

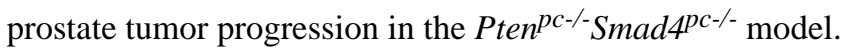




\section{Cxcl5-Cxcr2 signaling promotes MDSC recruitment and Cxcr2 inhibition delays tumor progression in Pten ${ }^{\text {pc-/-Smad4 }}{ }^{\text {pc-/- }}$ mice}

To elucidate the cellular origins and signaling molecules governing MDSC recruitment to prostate tumors, we incorporated $m T m G$ dual fluorescence reporter allele into the

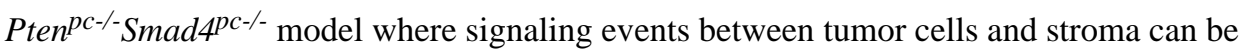
precisely delineated. The $m T m G$ allele (22) allows Cre-dependent GFP expression in prostate epithelial cells and ubiquitous tdTomato expression in all other non-Cre expressing cells (Figure 5A). Transcriptomic and IPA analyses of FACS-sorted $\mathrm{GFP}^{+}$tumor cells and Tomato $^{+}$stromal cells showed distinct expression patterns by hierarchical clustering (Figure 5A) with tumor cells enriched for pathways involved in cell adhesion molecules and tight junction (consistent with their epithelial nature) and stromal cells displaying activation of more diverse pathways involved in chronic inflammation, such as cytokine/cytokine receptor interaction, and chemokine, Jak-STAT, TCR, and BCR signaling $(\mathrm{p}<0.01$, data not shown). This result is consistent with the immuno- and histopathological analyses showing a massive infiltration of immune cells in the Pten ${ }^{\text {pc- }- \text { Smad }} 4^{\text {pc-/- }}$ tumors.

Employing this new model, we sought to identify genes that were upregulated in Pten $^{\text {pc-/-Smad }^{p c-/-} \text { cancer cells relative to Pten }}{ }^{\text {pc-/ }}$ cancer cells that might illuminate mechanisms involved in the recruitment of MDSCs by classifying the upregulated genes into either stroma- or tumor-enriched genes. To this end, our previously generated list of 242

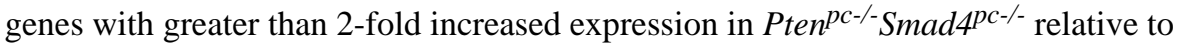
Pten $^{\text {pc-/- }}$ tumors (11) was intersected with 486 genes preferentially expressed in Pten $^{\text {pc-/-Smad4pc-/ }}$ GFP+ cancer cells relative to Tomato+ stroma cells (fold change $\geq 4$ ) (Supplementary Table S3 and S4), yielding 28 genes that are markedly enriched in

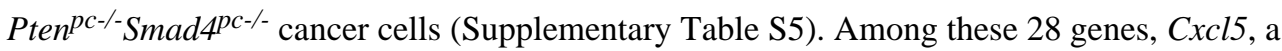
key cytokine involved in MDSC recruitment $(23,24)$, is the most significantly upregulated cancer cell-specific cytokine in Pten ${ }^{\text {pc-/-Smad }} 4^{\text {pc-/- }}$ tumors as compared to Pten ${ }^{\text {pc-/- tumors }}$ (Figure 5B and Supplementary Figure S5A). Notably Cxcr2, the cognate receptor for Cxc15, is also upregulated in Pten ${ }^{p c-/-S m a d 4 p c-/-}$ tumors as compared to Pten ${ }^{p c-/}$ tumors and is significantly enriched in Pten ${ }^{p c-/}$ Smad $4 p c-/-$ Tomato $^{+}$stroma cells (Figure 5B). The

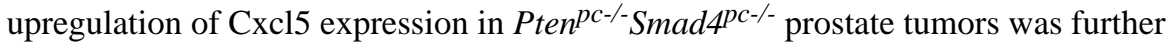
confirmed by IHC (Figure 5C). In addition, we performed FACS analysis of CD $11 \mathrm{~b}^{+} \mathrm{Gr}-1^{+}$ cells and $\mathrm{CD}_{11} \mathrm{~b}^{-} \mathrm{Gr}-1^{-}$cells from bone marrow, spleen, peripheral blood, and tumors for Cxcr2 expression. As shown in Supplementary Figure S5B, CD11 b- Gr-1 $1^{-}$cells (largely lymphocytes) are devoid of Cxcr2 expression, whereas a large fraction of $\mathrm{CD}_{11} \mathrm{~b}^{+} \mathrm{Gr}-\mathrm{1}^{+}$ cells express Cxcr2. When Cxcr2 expression was further separated into $\mathrm{Cxcr} 2^{\text {high }}$ and Cxcr2 $2^{+}$, we observed an enrichment of the $\mathrm{Cxcr} 2^{\text {high }}$ subpopulations in the CD11 b $\mathrm{b}^{+} \mathrm{Gr}-1^{+}$ cells in prostate tumor compared with $\mathrm{CD} 11 \mathrm{~b}^{+} \mathrm{Gr}-1^{+}$cells from bone marrow, spleen or blood (Supplementary Figure S5B). This is consistent with the model of active recruitment of MDSCs by tumors through Cxcr2-mediated chemoattraction.

To validate the Cxc15-Cxcr2 axis in the recruitment MDSCs to the TME of $\mathrm{Pten}^{\text {pc-/-Smad }} 4^{\text {pc-/- }}$ tumors, we assessed the impact of pharmacological inhibition of $\mathrm{Cxcl} 5$ and Cxcr2 in MDSCs using a transwell migration assay (23). First, anti-Cxc15 neutralizing

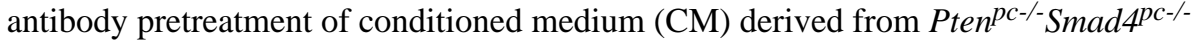


prostate cancer cell line resulted in decreased migration of MDSCs (Figure 5D); Second, Cxcr2 inhibitor SB255002 or anti-Cxcr2 neutralizing antibody pretreatment also impeded migration of MDSCs (Figure 5D); Third, in vivo blockade of the Cxcl5-Cxcr2 axis using SB255002 in 14-week Pten ${ }^{\text {pc-/-Smad4 }}{ }^{\text {pc-/- }}$ mice over a 14-day daily dosing schedule revealed a dramatic reduction in infiltration of MDSCs in the prostate tumors (Supplementary Figure S5C-D). Notably, similar to mice treated with anti-Gr-1 neutralizing

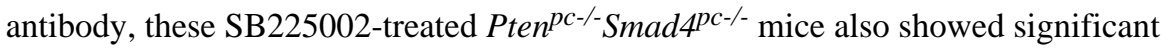
reduction in tumor burden (VP \& DLP) as compared to the vehicle-treated controls (Figure 5E \& Supplementary Figure S5E). Strikingly, all SB225002-treated tumors presented with PIN pathology while control group uniformly possessed advanced adenocarcinoma (Figure 5F \& Supplementary Table S2). Furthermore, SB225002 treatment significantly prolonged the overall survival of the Pten ${ }^{\text {pc-l-Smad4pc-/ }}$ mice as compared to the vehicle control (Figure 5G). Thus, we conclude that the Cxc15-Cxcr2 axis plays a prominent role in the

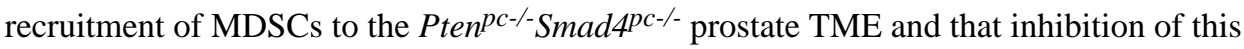
axis profoundly impairs tumor progression.

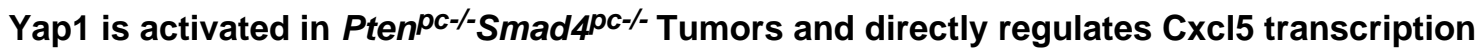

Having identified cancer cell-derived Cxcl5 as a key signaling molecule governing recruitment of MDSCs into the TME, we sought to define the molecular mechanisms

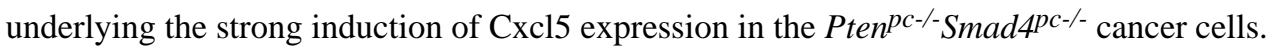
As Cxcl5 expression is not significantly upregulated in the Pten ${ }^{p c-/}$ tumors (Figure 5C), we performed unbiased Gene Set Enrichment Analysis (GSEA) to identify pathways that were activated in the Pten ${ }^{\text {pc-/-Smad4 } 4 \text { pc-/ }}$ tumors as compared to Pten ${ }^{\text {pc-/ }}$ tumors, aiming to identify potential regulators for $\mathrm{Cxcl5}$ in $\mathrm{Pten}^{p c-/} \mathrm{Smad}_{4} \mathrm{pc-l}^{-/}$tumors. The YAP oncogenic signature emerged as the second most hyperactivated pathway (Figure 6A and Supplementary Figure S6A). While it is known that the Hippo-YAP pathway plays an important role in development and cancer in organs such as liver, skin, intestine, pancreas (25-27), the role for Hippo-YAP pathway in prostate cancer biology is emerging. Specifically, Hippo pathway components LATS1/2 have been implicated in anoikis and metastasis in prostate cancer (28) and ERG-induced Yap1 activation can promote agerelated prostate tumor development (29). However, beyond the cancer cell specific functions, the Hippo-Yap1 pathway has not been linked to signaling communication between cancer cells and immune cells in the TME. Consistent with the in silico analysis, IHC analysis documented a dramatic increase in the nuclear localization of Yap1 in Pten $^{p c-/}$ Smad 4 pc-/- $^{-}$cancer cells as compared to Pten ${ }^{\text {pc-/ }}$ cancer cells (Figure 6B). As Yap1, a transcriptional coactivator and the downstream mediator of Hippo signaling, is regulated posttranscriptionally by either kinase-mediated degradation or cytoplasmic sequestration (25), our findings of increased nuclear localization of Yap1 is consistent with the hypothesis that Hippo-YAP pathway is activated in the Pten ${ }^{p c-/}$ Smad4 $4^{p c-/}$ tumors. In addition, unbiased oPOSSUM analysis (30) indicated that TEAD1, a member of the TEAD transcription factor family that is required for Yap1 function, ranked second among the top ten transcription factors with over-represented binding sites in the 70 cancer-specific genes that were upregulated in the Pten ${ }^{p c-/}$ Smad 4 pc-/- tumors as compared to the Pten $^{\text {pc-/ }}$ tumors ( $\geq 1.5$ fold, Z-Score=13.362, Supplementary Figure S6B-C), an observation reinforcing the relevance of the Hippo-YAP pathway. Furthermore, we identified 6 YAP/TEAD binding 
motifs in the promoter of Cxc15 gene (Supplementary Figure 6D), suggesting Yap1 could be directly involved in the recruitment of MDSCs through regulating Cxcl5 expression. This hypothesis was supported by chromatin immunoprecipitation (ChIP) assay showing that Yap1 binds to Cxcl5 promoter (Figure 6C), and that shRNA-mediated knockdown of Yap1

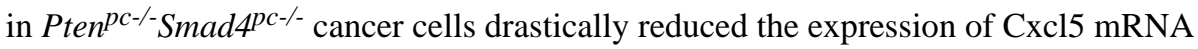
(Figure 6D). In addition, overexpression of a constitutively active Yap1 S127A mutant

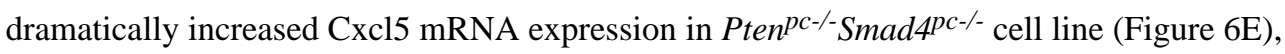
whereas overexpression of TEAD binding defective Yap1 mutant S127A/S94A compromised its ability to activate Cxcl5 transcription (Figure 6F). To examine the effect of Yap1-dependent cytokine signaling in the regulation of MDSCs recruitment, we first

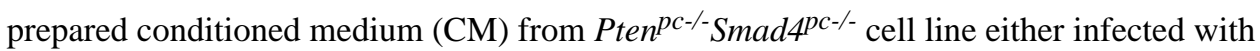
shRNA against Yap1 or pre-treated with Verteporfin (VP) (25), a small molecular inhibitor that disrupts Yap1-TEAD interaction. We then tested the effect of various CM on the migration of MDSCs in vitro. As shown in Figure 6G-H, we observed significantly decreased MDSCs migration in vitro when CM were from cells with either Yap1 knockdown or VP treatment.

Finally, to test if targeting Yap1 in vivo can impair the infiltration of MDSCs and inhibit tumor growth, we used our recently isolated syngeneic murine prostate cancer line PPS, which is derived from the backcrossed Pten ${ }^{p c-/}$ Smad4pc-/ $T p 53^{p c-/-}$ model (31) and can form subcutaneous or orthotopic tumors robustly in C57BL/6 hosts. Doxycycline-dependent shRNA knockdown of Yap1 (two independent shRNA designs \#1 and \#3) were established in PPS (Figure 6I), and injected subcutaneously in C57BL/6 mice. Yap1 knockdown induced by switching to doxycycline-containing drinking water resulted in a reduction of MDSCs in the intratumoral $\mathrm{CD}_{4} 5^{+}$population (Figure $6 \mathrm{~J}-\mathrm{K}$ ) and impaired tumor progression (Figure 6L). While the observation supports the hypothesis that targeting YAP1dependent MDSC infiltration impairs tumor growth, we acknowledge that the tumor growth impediment by Yap1 silencing is likely due to a combined effect of both cell nonautonomous and autonomous mechanisms. Together, these findings reveal a novel function for Yap1 in the recruitment of MDSCs through direct upregulation of Cxcl5 transcription in prostate tumor cells.

\section{YAP1 is activated in human prostate cancer and tracks with an MDSC signature}

To determine whether YAP1 is overexpressed and activated in human prostate cancer, we performed immunohistochemical staining of human prostate cancer tissue microarray (TMA) for YAP1. Interestingly, YAP1 is expressed in basal cells, but not in the luminal cells of the normal human prostate (Figure 7A). In addition, we observed YAP1 is overexpressed in a subset of human prostate cancers (Figure 7A-B \& Supplementary Table S6), consistent with a recent report (29). Given the lack of validated antibodies for human MDSCs for TMA analysis, we generated a list of 39 MDSC related genes curated from literature analysis (Supplementary Table S7) to generate evidence of a link between YAP1 activation and MDSC prominence in human prostate. Using the prostate TCGA RNA-seq data, unsupervised clustering with the 39-gene MDSC signature categorized 498 TCGA primary prostate tumors into three subtypes: MDSC-high ( $\mathrm{n}=139)$, MDSC-medium ( $\mathrm{n}=158)$, and MDSC-low ( $\mathrm{n}=201)$ (Figure 7C), suggesting that a subset of human prostate tumors 
may have prominent infiltration of MDSCs. In addition, using GSEA, we found that several YAP1 signature genes are significantly overexpressed in MDSC-high samples as compared to MDSC-low samples (Figure 7D; p value < 0.005), reinforcing the link between MDSChigh prostate tumors and YAP1 transcriptional activities. Furthermore, CXCL6, the human homologue of murine Cxc15, is expressed at a higher levels in the MDSC-high samples as compared to MDSC-low samples (Figure 7E, p=9.40E-29). Similar analysis was performed in a published dataset focused on tumor immunobiological differences in prostate cancer between African-American and European-American men (32). The 39-gene MDSC signature can cluster the 69 primary prostate tumors into MDSC-high $(\mathrm{n}=40)$ and MDSClow $(n=29)$ and Yap1 signatures were prominent in the MDSC-high groups (Supplementary Figure 7A-B). Together, these human prostate tumor findings, which parallel our murine observations, suggests that activated YAP1 is integral to MDSCs infiltration in both mouse and human prostate cancer, thus enhancing the translational value of the study.

\section{Discussion}

Although a large number of studies have demonstrated a direct relationship between MDSC frequency and tumor burden (5), our understanding of the role of MDSCs in tumor progression, particularly prostate cancer, remains largely speculative. Here, using a highly invasive Pten/Smad4 deficient prostate cancer model, we established the signaling circuits involved in the recruitment of MDSCs to the TME and demonstrated a critical role of these cells in facilitating tumor progression.

Homozygous deletion of Pten in murine prostate elicited a strong senescence response that restricts tumor progression (33), thus Pten-deficient prostate tumors are largely indolent and progress slowly to invasive prostate adenocarcinoma without metastasis to distant organs $(11,33)$. Recently, it was shown that infiltrating Gr- $1^{+}$myeloid cells suppress Pten lossinduced cellular senescence through a paracrine signaling mediated by myeloid secreted Il1ra (10). We have previously reported that deletion of Smad4 leads to bypass of Pten lossinduced senescence in PCa progression, resulting in aggressive cancer cell proliferation and invasion/metastasis (11). Using the state-of-the-art CyTOF technology, we reveal that progression in the Pten ${ }^{\text {pc-/-Smad }} 4^{p c-/}$ model is associated with abundant immune cell infiltration characterized by prominent representation of $\mathrm{CD} 11 \mathrm{~b}^{+} \mathrm{Gr}-1^{+} \mathrm{MDSCs}$, which display potent immunosuppressive activities as shown by their strong antagonistic effect on $\mathrm{T}$ cell proliferation (Figure 3A-B).

The basis for the increased frequency of MDSCs in the TME and, specifically in

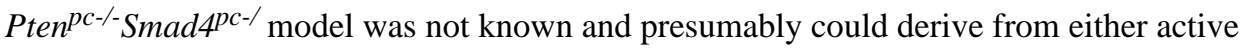
chemoattraction or passive nonspecific responses to tissue stress associated with expanding tumor burden. Taking an unbiased approach to identify pathways that may recruit MDSCs, we deconvoluted cancer versus stromal cell transcriptomes by exploiting a Cre-dependent dual fluorescence lineage tracing system in the Pten ${ }^{p c-/}$ Smad $4^{p c-/}$ model. This approach identified unique immune regulatory molecules that are activated prominently in Pten ${ }^{p c-/}$ Smad $4{ }^{p c-/}$ cancer cells, most prominently Cxcl5. We established that the Cxcl5 chemokine plays a key role in the efficient recruitment of MDSCs which enables tumor progression, as blocking Cxc15-Cxcr2 signaling with a Cxcr2 inhibitor led to reduced MDSC 
infiltration with associated anti-tumor effect. It should be noted that the human homolog for murine Cxc15 is CXCL6, and CXCL6 has been shown to be upregulated in prostate cancer as compared to normal prostate and significantly associated with high Gleason score 8-9 (34). Interestingly, it was shown that Cxcl5 promotes recruitment of MDSCs to the primary melanoma, resulting in epithelial-mesenchymal transition (EMT) and cancer cell dissemination (35). Thus, the possible role of Cxcl5/CXCL6 in prostate cancer metastasis merits further study.

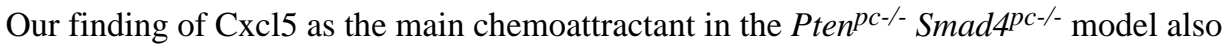
provided a framework to determine the cancer cell signaling pathways driving $\mathrm{Cxcl5}$ upregulation. By integration of bioinformatic analysis and experimental validation, we identified that YAP1 is activated in Pten ${ }^{\text {pc- }}$-Smad4pc-/- prostate tumors and that Yap1 directly regulates Cxcl5 transcription and MDSCs recruitment. In addition, we showed that YAP1 is overexpressed in a subset of human prostate cancers, which is consistent with a recent publication showing a correlation of ERG and YAP1 co-expressed in a subset of human prostate cancers (29). Importantly, a 39-gene MDSCs signature clusters the prostate TCGA samples into three subtypes. By comparing the samples with high and low abundance of MDSC-related gene expression, YAP1 signatures and higher expression of CXCL6 are identified in the MDSC-high samples, which is consistent with our findings in the mouse model. Furthermore, the 39-MDSC gene signature can cluster primary prostate tumor samples from a published dataset (32) into two subtypes using MDSC-high and MDSC-low, with Yap1 signatures identified in the MDSC-high subtype. The Hippo-YAP signaling pathway is widely deregulated in human solid neoplasia and often associated with enhanced cancer cell proliferation and cancer stem cell phenotypes (25), and is implicated in the regulation of anoikis and metastasis in prostate cancer (28) and the development of agerelated prostate cancers driven by ERG overexpression (29), yet how Hippo-YAP pathway regulates the TME in prostate cancer has hitherto not yet been elucidated. Our finding of a novel non-cell-autonomous function for Hippo-YAP signaling in MDSC recruitment in TME complements well the recently elucidated roles of YAP1 in promoting cell autonomous functionality of cancer cells, including enhanced tumor survival, EMT and bypass mechanism for oncogene addiction $(26,27)$.

Pharmacological depletion of MDSCs using Gr-1 antibody, Pep-H6 peptibody, or Cxcr2 inhibitor arrested prostate progression at the high-grade PIN stage while controls exhibited full-fledged adenocarcinoma in Pten ${ }^{p c-/}$ Smad $4^{\text {pc-/ }}$ model. Given that treatment commences at 14 weeks of age (Supplementary Figure S3A), when prostate tumors have uniformly advanced to the invasive adenocarcinoma stage (11) with significant MDSC infiltration (Figure 2B), our findings support the view that anti-MDSC treatment provokes regression of advanced tumors. In addition, both Pep-H6 peptibody and Cxcr2 inhibitor treatment significantly prolonged the overall survival of the $\mathrm{Pten}^{\text {pc-/-Smad }}{ }^{\text {pc-/- }}$ tumor-bearing mice. Therefore, our preclinical data suggests that pharmacological depletion of MDSCs may offer potential therapeutic benefits for advanced prostate cancer patients, particularly those deficient for PTEN and SMAD4. In line with our findings, others have demonstrated that depletion of G-MDSCs promotes the intratumoral accumulation of activated CD8 T cells and apoptosis of tumor epithelial cells in a Kras/Trp53 mouse pancreatic cancer model (36). 
MDSCs are of myeloid cell lineage, and their coordinated regulation represents one of the most complex aspects of cancer-host interactions (37). The involvement of the myeloid compartment of the hematopoietic system in innate immunity, adaptive immunity as well as in regulation of TME through non-immune mechanisms, highlights the need to understand more deeply how modulating different myeloid populations, including MDSCs, can positively or negatively affect tumor growth.

Pep-H6 peptibody, targeting S100a9 expressed on MDSCs, has been shown to have minimal toxicity in treated mice (20) and potent anti-tumor activity (Figure 4D-E \& Ref 18). Interestingly, Tasquinimod, a small molecular inhibitor for S100A9, has been shown to increase progression-free survival (PFS) and overall survival (OS) for metastatic castration resistant prostate cancer (mCRPC) in phase II clinical trial and has entered phase III clinical trial (38). Importantly, similar to the peptibody treatment in mice, Tasquinimod is welltolerated and only causes minor adverse effects in human patients (38), suggesting that Tasquinimod or similar drugs that targeting S100A9 could be potentially used as chemopreventive agent for patients with high risk primary prostate cancer. The antiproliferative mechanism may explain why targeting Cxcr2 in PCa with abundant preexisting MDSC infiltration can lead to MDSC depletion, as MDSCs have been shown to undergo active proliferation inside the prostate tumor of the Pten ${ }^{\text {pc- }}$ model (9). The effectiveness of targeting Cxcr2 in our model suggests targeting mechanisms that specifically regulate MDSC recruitment as well as their proliferative and survival potential in human cancers would provide therapeutic benefit for patients with PCa.

Targeting MDSCs as a cooperative approach for immunotherapy is clinically relevant, as increasing evidence indicates MDSCs represent a bona fide immunosuppressive cell population in patients with various solid tumors $(39,40)$. Immunosuppressive mechanisms by MDSCs in mice have been validated in humans, which include L-arginine depletion, NO and ROS production, TGF $\beta$ secretion, blocking $T_{\text {eff }}$ cells and inducing $T_{\text {reg }}$ cells, among others (39). Future studies are warranted to evaluate if combining MDSC depletion with immune checkpoint inhibitors such as anti-CTLA4, anti-PD1 and anti-PD-L1 antibodies may elicit synergistic efficacy in the preclinical models of PCa and eventually benefit patients with prostate cancer.

\section{Methods}

\section{Mice strains}

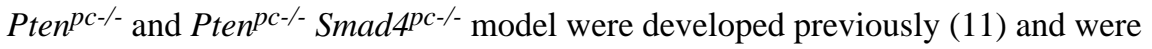
backcrossed to the C57BL/6 background for more than 4 generations. B6.129(Cg)Gt(ROSA)26Sortm4(ACTB-tdTomato,-EGFP)Luo/J (“ $m$ TmG”) strain was obtained from Jackson Laboratory. Mice were maintained in pathogen-free conditions at M.D. Anderson Cancer Center. All manipulations were approved under MD Anderson Cancer Center (MDACC) Institutional Animal Care and Use Committee (IACUC). 


\section{Cell lines}

Pten ${ }^{p c-/}$-Smad4pc-/ prostate cell lines have been described previously (11), which was generated in 2010. PPS, a C57BL/6-syngeneic cell line isolated from prostate tumor of Pten $^{p c-/}$ Smad4 $4^{p c-/} T p 53^{c-/}$ mice was generated in 2013. All cell lines tested for Mycoplasma were negative within 6 months of performing the experiments. Cell line authentication was not performed.

\section{CyTOF and Flow cytometry}

Prostate tumor single cells were isolated using the Mouse Tumor Dissociation kit (Miltenyl Biotec). Single cells were isolated from spleen, lymph node, and peripheral blood using standard protocol. All isolated cells were depleted of erythrocytes by hypotonic lysis. For CyTOF analysis, cells were blocked for Fc $\gamma \mathrm{R}$ using CD16/CD32 antibody (clone 2.4G2, BD Biosciences) and incubated with CyTOF antibody mix for 30 minutes at room temperature. Cells were washed once and incubated with MAXPAR®Nucleic Acid Intercalator- ${ }^{103} \mathrm{Rh}$ (DVS Sciences) for 20 minutes for viability staining. Cells were fixed with $1.6 \%$ formaldehyde for 1 hour and incubated with MAXPAR®Nucleic Acid Intercalator-Ir (DVS Sciences) at $4^{\circ} \mathrm{C}$ overnight to stain the nuclei. The samples were analyzed with CyTOF instrument (DVS Sciences) in the Flow Cytometry and Cellular Imaging Core Facility at M.D. Anderson Cancer Center. Flow cytometry were performed using standard protocol on LSRFortessa analyzer (Becton Dickinson) and analyzed with FlowJo software (Tree Star).

\section{T cell suppression and MDSCs migration assay}

T cell suppression assay was performed as described (9) using FACS-sorted MDSCs and CFSE (Invitrogen) labeled MACS-sorted (Miltenyi) $\mathrm{CD} 8^{+}$or $\mathrm{CD} 4^{+} \mathrm{T}$ cells in anti-CD3 and anti-CD28 coated 96-well plates at an MDSC/T cell ratio of 0:1, 1:1, 1:2, 1:4, with $3.0 \times 10^{5}$ to $5.0 \times 10^{5}$ MDSCs used in each ratio. Cells were analyzed after 72 hours by Flow cytometry and the suppression of T cells is calculated as described (41). The percentage of CFSE+ cells divided in the presence of MDSCs was compared to percentage of CFSE+ divided cells in the absence of any added MDSCs. For MDSCs migration assay, equal number of FACS-sorted MDSCs, untreated or pre-treated with neutralizing antibody or inhibitor, were placed on the upper chamber of a transwell system (BD Falcon) and conditioned media (CM) from Pten/Smad4-deficient cells under various condition were added to the bottom chamber. Cells were allowed to migrate to the bottom well for 6 hours at $37{ }^{\circ} \mathrm{C}, 5 \% \mathrm{CO}_{2}$. Migrated cells were then analyzed by flow cytometry using BD Fortessa X20. Migrated FITC positive cells were gated to count the absolute number of cells migrated through the transwell.

\section{MSDCs depletion in vivo with Gr1 antibody, Peptibody, and Cxcr2 inhibitor SB225002}

Anti-Gr-1 (clone RB6-8C5) and isotype control (clone LTF-2) were purchased from BioXcell and dosed at $200 \mu \mathrm{g} / \mathrm{mouse}$ (i.p.) every other day. $15 \mu \mathrm{g}$ of endotoxin-free plasmids for irrelevant control peptibody (Irr-pep) and MDSC-specific Pep-H6 peptibody were injected into mice through tail vein using the established protocol (21) in TransIT-EE Delivery Solution (Mirus Bio LLC) every 4 days. SB225002 (Cayman Chemical) in DMSO 
was diluted in vehicle $(0.9 \% \mathrm{NaCl}, 0.3 \%$ Tween 80$)$ for in vivo administration every other day $(5 \mathrm{mg} / \mathrm{kg})$.

\section{Inducible Yap1 knockdown}

Inducible Yap1 knockdown was constructed by cloning the two Yap1 shRNA used previously (26) from the pLKO.1 into a doxycycline inducible plasmid. Lentivirus was packaged in 293T and was used to infect PPS, a C57BL/6-syngeneic cell line isolated from

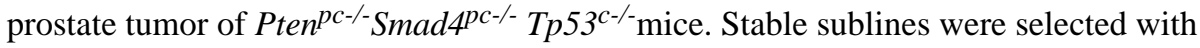
puromycine $(2 \mu \mathrm{g} / \mathrm{mL})$ and injected subcutaneously to the flank of 5-week old male C57BL/6 mice (Jackson Laboratory). Two weeks post-injection, mice were fed with doxycycline water $(2 \mathrm{~g} / \mathrm{L})$, a method used to execute doxycycline inducible expression in vivo (42). Tumors were measured and extracted 6 days later to analyze for MDSC\% in infiltrating immune cells.

\section{Computational analysis of mouse microarray data and human prostate TCGA data}

RNA was isolated from FACS-sorted $\mathrm{GFP}^{+}$and Tomato $^{+}$cells using

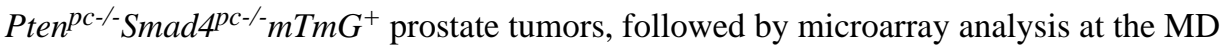
Anderson Microarray Core facility using the Mouse Genome 430 2.0 Array (Affymetrix) to

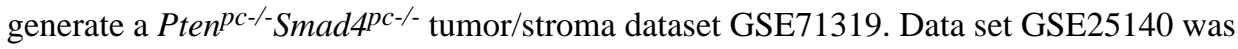
downloaded from NCBI GEO database. Differentially expressed genes between two conditions (GFP+ vs. Tomato+, or Pten/Smad4 vs. Pten) were subjected to IPA, GSEA, and oPOSSUM analysis. For analysis of human prostate data, we first generated a list of 39 human MDSCs signature genes by literature mining (Supplementary Table S7). The gene expression data of 498 TCGA prostate samples was downloaded from the Broad GDAC Firehose (http://gdac.broadinstitute.org), which is the RSEM expression estimates normalized to set the upper quartile count at 1000 for gene level and then with $\log 2$ transformation. The 498 TCGA prostate samples were clustered using the 39 MDSC genes into MDSC-high, MDSC-low, and MDSC-medium (distance between pairs of samples were measured by manhattan distance, and clustering was then performed using complete-linkage hierarchical clustering). 69 samples from Wallace et al (32) were cluster into MDSC-high and MDSC-low. Differentially expressed genes between MDSC- high and MDSC-low were analyzed by GSEA. The expression of CXCL6 in MDSC-high samples is compared to MDSC-low samples using wilcoxon test.

\section{Immunohistochemistry and Western Blot Analysis}

Tissues were fixed in 10\% formalin overnight and embedded in paraffin. Immunohistochemical (IHC) was performed as described earlier (11). For western blot analysis, cells were lysed on ice using RIPA buffer (Boston BioProducts) supplemented with protease and phosphatase inhibitors (Roche). YAP1 antibody was obtained from Novus Bio and Cell Signaling Technology. Cxc15 antibodies were obtained from Bioss (Woburn, MA) and R\&D Biosystems (Minneaplois, MN). Cxcr2 antibody was obtained from Bioss and R $\&$ D Biosystems. CD45 and Ly-6G antibodies were obtained from Biolegend. Prostate tissue micrcroarray was obtained from Folio Bioscience. 


\section{Chromatin immunoprecipitation}

Chromatin immunoprecipitation (ChIP) was performed as described (26) using YAP1 antibody from Novus. Briefly, 5ug of Rabbit IgG (Santa Cruz) or YAP1 antibody was incubate with Protein A Dynabeads magnetic beads (Invitrogen) for 4 hours, followed by extensive wash to remove unbound antibody. Antibody-Beads were then added to the chromatin and incubate for overnight. The following Primers were used for qPCR analysis: Cxc15_S: 5'-CTCCAGTTTCCTGCCTGAAG-3' and Cxc15_as: 5'GTGTGGAGATTGGGGCTCTA-3'.

\section{Quantitative RT-PCR}

RNA was isolated by RNeasy Kit (Qiagen) and reversed transcribed using Superscript III cDNA synthesis Kit (Life Technology). Quantitative PCR was performed using SYBRGreenER Kit (Life Technology). The following primers were used: Cxcl5_Fwd: GCATTTCTGTTGCTGTTCACGCTG, Cxc15_Rev: CCTCCTTCTGGTTTTTCAGTTTAGC; $\beta$-actin_Fwd: GAAATCGTGCGTGACATCAAAG, $\beta$-actin_Rev: TGTAGTTTCATGGATGCCACAG; Yap1_Fwd: TGAGATCCCTGATGATGTACCAC, Yap1_Rev: TGTTGTTGTCTGATCGTTGTGAT.

\section{Statistical Analysis}

Data are presented as mean \pm SD unless indicated otherwise. Student's $t$ test assuming twotailed distributions was used to calculate statistical significance between groups. Animal survival benefit was determined by Kaplan-Meier analysis. $P<0.05$ was considered statistically significant.

\section{Supplementary Material}

Refer to Web version on PubMed Central for supplementary material.

\section{Acknowledgments}

We thank Dr. Larry W Kwak and Dr. Hong Qin for the peptibody plasmids; Dr. Willem Overwijk and Dr. Yared Hailemichael for advise in hydrodynamic injection; Samirkumar Amin and member of the DePinho laboratory for helpful suggestions and technical support; Flow Cytometry and Cellular Imaging Core Facility (Jared Burks, Duncan Mak, and Karen Dwyer), Sequencing \& Non-coding RNA Core Services (Chang-Gong Liu) at The University of Texas, MD Anderson Cancer Center (Cancer Center Support grant, CA16672).

Grant Support: The project was supported by U01CA141508 (L.C. and R.A.D.), Prostate Cancer Research Program (PCRP) W81XWH-13-1-0202 (G.W.), W81XWH-14-1-0429 (P.D.), Idea Development Award - New Investigator Option (W81XWH-14-1-0576) (X.L.) from Department of Defense, and Clayton \& Modesta Williams Cancer Research Fund. Additional support was provided by Jane Coffin Childs Memorial Fund Postdoctoral Fellowship to X.L. E.J. is supported by Korean Governments (MISP) grand number 2011-0030130.

\section{References}

1. Karlou M, Tzelepi V, Efstathiou E. Therapeutic targeting of the prostate cancer microenvironment. Nat Rev Urol. 2010; 7:494-509. [PubMed: 20818327]

2. Junttila MR, de Sauvage FJ. Influence of tumour micro-environment heterogeneity on therapeutic response. Nature. 2013; 501:346-54. [PubMed: 24048067] 
3. Hanahan D, Coussens L. Accessories to the Crime: Functions of Cells Recruited to the Tumor Microenvironment. Cancer Cell. 2012; 21:309-22. [PubMed: 22439926]

4. Hanahan D, Weinberg Robert A. Hallmarks of Cancer: The Next Generation. Cell. 2011; 144:64674. [PubMed: 21376230]

5. Talmadge JE, Gabrilovich DI. History of myeloid-derived suppressor cells. Nat Rev Cancer. 2013; 13:739-52. [PubMed: 24060865]

6. Vuk-Pavlović S, Bulur PA, Lin Y, Qin R, Szumlanski CL, Zhao X, et al. Immunosuppressive CD14+HLA-DRlow/- monocytes in prostate cancer. Prostate. 2010; 70:443-55. [PubMed: 19902470]

7. Idorn M, Kollgaard T, Kongsted P, Sengelov L, Thor Straten P. Correlation between frequencies of blood monocytic myeloid-derived suppressor cells, regulatory $\mathrm{T}$ cells and negative prognostic markers in patients with castration-resistant metastatic prostate cancer. Cancer immunology, immunotherapy : CII. 2014; 63:1177-87. [PubMed: 25085000]

8. Brusa D, Simone M, Gontero P, Spadi R, Racca P, Micari J, et al. Circulating immunosuppressive cells of prostate cancer patients before and after radical prostatectomy: profile comparison. Int $\mathrm{J}$ Urol. 2013; 20:971-8. [PubMed: 23421558]

9. Garcia AJ, Ruscetti M, Arenzana TL, Tran LM, Bianci-Frias D, Sybert E, et al. Pten null prostate epithelium promotes localized myeloid-derived suppressor cell expansion and immune suppression during tumor initiation and progression. Molecular and cellular biology. 2014; 34:2017-28. [PubMed: 24662052]

10. Di Mitri D, Toso A, Chen JJ, Sarti M, Pinton S, Jost TR, et al. Tumour-infiltrating Gr-1+ myeloid cells antagonize senescence in cancer. Nature. 2014; 515:134-7. [PubMed: 25156255]

11. Ding Z, Wu CJ, Chu GC, Xiao Y, Ho D, Zhang J, et al. SMAD4-dependent barrier constrains prostate cancer growth and metastatic progression. Nature. 2011; 470:269-73. [PubMed: 21289624]

12. Bjornson ZB, Nolan GP, Fantl WJ. Single-cell mass cytometry for analysis of immune system functional states. Current opinion in immunology. 2013; 25:484-94. [PubMed: 23999316]

13. Youn JI, Nagaraj S, Collazo M, Gabrilovich DI. Subsets of myeloid-derived suppressor cells in tumor-bearing mice. Journal of Immunology. 2008; 181:5791-802.

14. Kusmartsev S, Nefedova Y, Yoder D, Gabrilovich DI. Antigen-specific inhibition of CD8+ T cell response by immature myeloid cells in cancer is mediated by reactive oxygen species. J Immunol. 2004; 172:989-99. [PubMed: 14707072]

15. Szuster-Ciesielska A, Hryciuk-Umer E, Stepulak A, Kupisz K, Kandefer-Szerszen M. Reactive oxygen species production by blood neutrophils of patients with laryngeal carcinoma and antioxidative enzyme activity in their blood. Acta Oncol. 2004; 43:252-8. [PubMed: 15244248]

16. Schmielau J, Finn OJ. Activated granulocytes and granulocyte-derived hydrogen peroxide are the underlying mechanism of suppression of T-cell function in advanced cancer patients. Cancer Res. 2001; 61:4756-60. [PubMed: 11406548]

17. Pekarek LA, Starr BA, Toledano AY, Schreiber H. Inhibition of tumor growth by elimination of granulocytes. The Journal of experimental medicine. 1995; 181:435-40. [PubMed: 7807024]

18. Wang G, Lunardi A, Zhang J, Chen Z, Ala U, Webster KA, et al. Zbtb7a suppresses prostate cancer through repression of a Sox9-dependent pathway for cellular senescence bypass and tumor invasion. Nat Genet. 2013; 45:739-46. [PubMed: 23727861]

19. Lunardi A, Ala U, Epping MT, Salmena L, Clohessy JG, Webster KA, et al. A co-clinical approach identifies mechanisms and potential therapies for androgen deprivation resistance in prostate cancer. Nat Genet. 2013; 45:747-55. [PubMed: 23727860]

20. Qin H, Lerman B, Sakamaki I, Wei G, Cha SC, Rao SS, et al. Generation of a new therapeutic peptide that depletes myeloid-derived suppressor cells in tumor-bearing mice. Nat Med. 2014; 20:676-81. [PubMed: 24859530]

21. Liu F, Song Y, Liu D. Hydrodynamics-based transfection in animals by systemic administration of plasmid DNA. Gene therapy. 1999; 6:1258-66. [PubMed: 10455434]

22. Muzumdar MD, Tasic B, Miyamichi K, Li L, Luo L. A global double-fluorescent Cre reporter mouse. Genesis. 2007; 45:593-605. [PubMed: 17868096] 
23. Yang L, Huang J, Ren X, Gorska AE, Chytil A, Aakre M, et al. Abrogation of TGF beta signaling in mammary carcinomas recruits $\mathrm{Gr}-1+\mathrm{CD} 11 \mathrm{~b}+$ myeloid cells that promote metastasis. Cancer Cell. 2008; 13:23-35. [PubMed: 18167337]

24. Bierie B, Stover DG, Abel TW, Chytil A, Gorska AE, Aakre M, et al. Transforming growth factorbeta regulates mammary carcinoma cell survival and interaction with the adjacent microenvironment. Cancer Res. 2008; 68:1809-19. [PubMed: 18339861]

25. Johnson R, Halder G. The two faces of Hippo: targeting the Hippo pathway for regenerative medicine and cancer treatment. Nature reviews Drug discovery. 2014; 13:63-79. [PubMed: 24336504]

26. Kapoor A, Yao W, Ying H, Hua S, Liewen A, Wang Q, et al. Yap1 activation enables bypass of oncogenic Kras addiction in pancreatic cancer. Cell. 2014; 158:185-97. [PubMed: 24954535]

27. Shao DD, Xue W, Krall EB, Bhutkar A, Piccioni F, Wang X, et al. KRAS and YAP1 converge to regulate EMT and tumor survival. Cell. 2014; 158:171-84. [PubMed: 24954536]

28. Zhao B, Li L, Wang L, Wang CY, Yu J, Guan KL. Cell detachment activates the Hippo pathway via cytoskeleton reorganization to induce anoikis. Genes \& development. 2012; 26:54-68. [PubMed: 22215811]

29. Nguyen LT, Tretiakova MS, Silvis MR, Lucas J, Klezovitch O, Coleman I, et al. ERG Activates the YAP1 Transcriptional Program and Induces the Development of Age-Related Prostate Tumors. Cancer Cell. 2015; 27:797-808. [PubMed: 26058078]

30. Kwon AT, Arenillas DJ, Worsley Hunt R, Wasserman WW. oPOSSUM-3: advanced analysis of regulatory motif over-representation across genes or ChIP-Seq datasets. G. 2012; 32:987-1002.

31. Ding Z, Wu CJ, Jaskelioff M, Ivanova E, Kost-Alimova M, Protopopov A, et al. Telomerase Reactivation following Telomere Dysfunction Yields Murine Prostate Tumors with Bone Metastases. Cell. 2012; 148:896-907. [PubMed: 22341455]

32. Wallace TA, Prueitt RL, Yi M, Howe TM, Gillespie JW, Yfantis HG, et al. Tumor immunobiological differences in prostate cancer between African-American and EuropeanAmerican men. Cancer Res. 2008; 68:927-36. [PubMed: 18245496]

33. Chen Z, Trotman LC, Shaffer D, Lin HK, Dotan ZA, Niki M, et al. Crucial role of p53-dependent cellular senescence in suppression of Pten-deficient tumorigenesis. Nature. 2005; 436:725-30. [PubMed: 16079851]

34. Liu Q, Russell MR, Shahriari K, Jernigan DL, Lioni MI, Garcia FU, et al. Interleukin-1beta promotes skeletal colonization and progression of metastatic prostate cancer cells with neuroendocrine features. Cancer Res. 2013; 73:3297-305. [PubMed: 23536554]

35. Toh B, Wang X, Keeble J, Sim WJ, Khoo K, Wong WC, et al. Mesenchymal transition and dissemination of cancer cells is driven by myeloid-derived suppressor cells infiltrating the primary tumor. PLoS Biol. 2011; 9:e1001162. [PubMed: 21980263]

36. Stromnes IM, Brockenbrough JS, Izeradjene K, Carlson MA, Cuevas C, Simmons RM, et al. Targeted depletion of an MDSC subset unmasks pancreatic ductal adenocarcinoma to adaptive immunity. Gut. 2014; 63:1769-81. [PubMed: 24555999]

37. Gabrilovich DI, Ostrand-Rosenberg S, Bronte V. Coordinated regulation of myeloid cells by tumours. Nature Reviews Immunology. 2012; 12:253-68.

38. Osanto S, van Poppel H, Burggraaf J. Tasquinimod: a novel drug in advanced prostate cancer. Future oncology. 2013; 9:1271-81. [PubMed: 23980674]

39. Filipazzi P, Huber V, Rivoltini L. Phenotype, function and clinical implications of myeloid-derived suppressor cells in cancer patients. Cancer Immunology, Immunotherapy. 2012; 61:255-63. [PubMed: 22120756]

40. Solito S, Marigo I, Pinton L, Damuzzo V, Mandruzzato S, Bronte V. Myeloid-derived suppressor cell heterogeneity in human cancers. Annals of the New York Academy of Sciences. 2014; 1319:47-65. [PubMed: 24965257]

41. Battaglia M, Stabilini A, Roncarolo MG. Rapamycin selectively expands CD4+CD25+FoxP3+ regulatory T cells. Blood. 2005; 105:4743-8. [PubMed: 15746082]

42. Ying H, Kimmelman AC, Lyssiotis CA, Hua S, Chu GC, Fletcher-Sananikone E, et al. Oncogenic Kras maintains pancreatic tumors through regulation of anabolic glucose metabolism. Cell. 2012; 149:656-70. [PubMed: 22541435] 
A

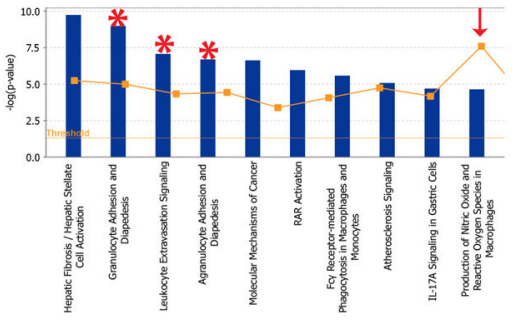

VP

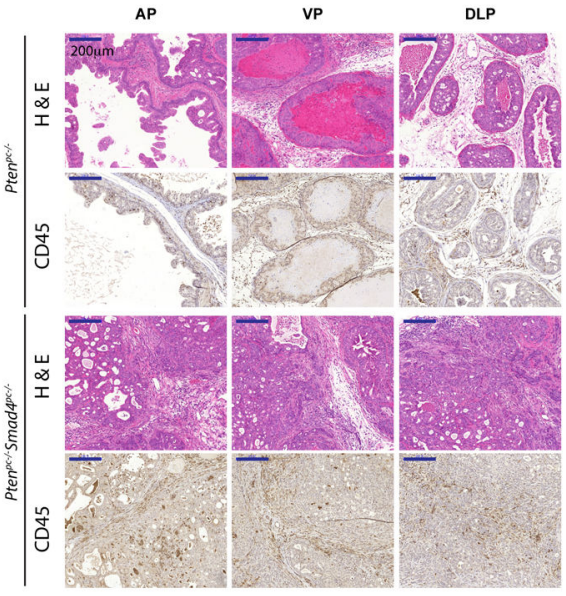

C

D
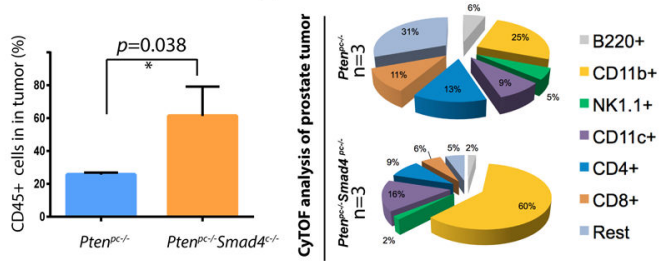

Figure 1. Prominent Infiltration of Immune Cells in the Pten ${ }^{\text {pc-/-Smad4 }}{ }^{\text {c-/- }}$ Tumors as Compared to Pten $^{\text {pc-/- }}$ Tumors

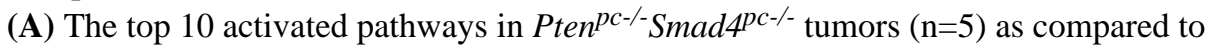
Pten $^{\text {pc-/- }}$ tumors $(\mathrm{n}=5)$ identified by Ingenuity pathway analysis (IPA). (B) A significant

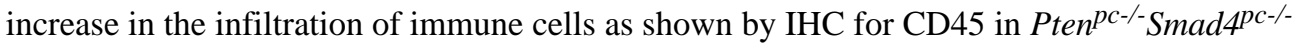
tumors as compared to Pten ${ }^{\text {pc-/- }}$ tumors from 16 weeks old mice $(\mathrm{n}=3)$. AP, VP and DLP stand for anterior prostate, ventral prostate and dorsolateral prostate, respectively. Scale bar $200 \mu \mathrm{m}$. (C) Quantification of tumor-infiltrating CD45 ${ }^{+}$cells (AP, VP and DLP combined) in Pten ${ }^{\text {pc-/- }}$ tumors and Pten ${ }^{\text {pc-/-Smad4 }}$ Pc-/- $^{\text {from } 16}$ weeks old mice $(\mathrm{n}=3)$, assessed by CyTOF. (D) Percentages of various immune cell populations within the CD $45^{+}$infiltrating immune cells in prostate tumors from 16 weeks old Pten ${ }^{\text {pc-/- }}$ and Pten ${ }^{\text {pc-/-Smad4pc-/- }}$ mice, assessed with CyTOF (9-marker) and analyzed with Flowjo. CD11b+ myeloid cells are significantly more in Pten ${ }^{p c-/}$ Smad4 ${ }^{\text {pc-/- }}$ tumor as compared to Pten ${ }^{\text {pc-/- }}$ tumor $(\mathrm{n}=3$, $P<0.05)$. 
A
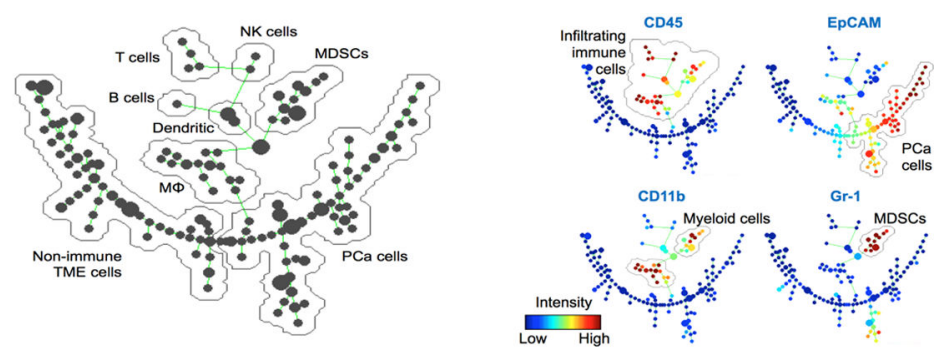

B

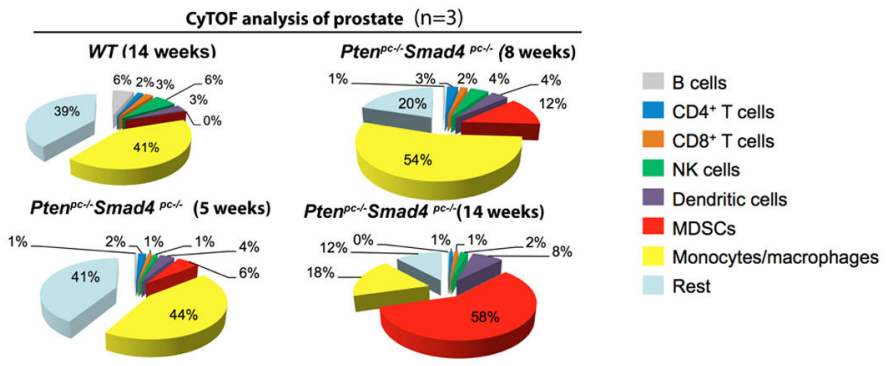

C

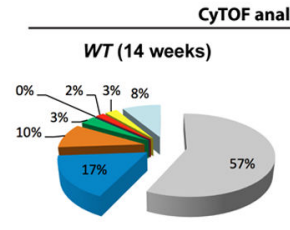

Pten ${ }^{\text {pc/-Smad4 }}{ }^{\text {per- }}$ (5 weeks)

$14 \%$

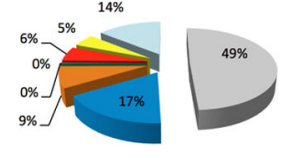

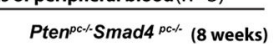

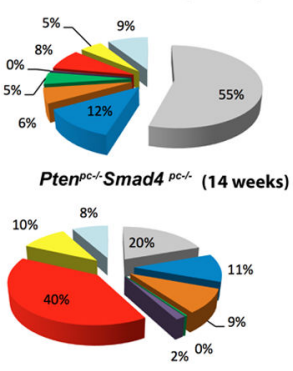

B cells

$\square \mathrm{CD} 4^{+} \mathrm{T}$ cells

CD8 ${ }^{+} T$ cells

NK cells

Dendritic cells

MDSCs

Monocytes/macrophages

Rest

Figure 2.

$\mathrm{CD}_{11 \mathrm{~b}^{+}} \mathrm{Gr}_{-1} \mathbf{1}^{+}$Cells are Significantly Increased in Pten $^{\text {pc-/- }}$ Smad4pc-/- Tumors as

Compared to Pten $^{\text {pc-/- }}$ Tumors. (A) SPADE tree derived from CyTOF (17-marker)

analysis of whole tumor cell population from $\mathrm{Pten}^{\text {pc-/-Smad4 }}{ }^{\text {pc-/- }}$ mice at 5-week, 8-week, and 14-week old $(n=3)$. Live single cells were used to construct the tree. Cell populations were identified as PCa cells (EpCAM ${ }^{+} \mathrm{CD}^{-} 5^{-}$), non-immune TME cells (EpCAM- $\left.\mathrm{CD}^{-} 5^{-}\right)$, $\mathrm{T}$ cells $\left(\mathrm{CD} 45^{+} \mathrm{CD}^{+} \mathrm{TCR}^{+}\right), \mathrm{B}$ cells $\left(\mathrm{CD} 45^{+} \mathrm{B} 220+\mathrm{CD} 19+\right)$, NK cells $\left(\mathrm{CD} 45^{+} \mathrm{NK} 1.1^{+}\right)$, dendritic cells $\left(\mathrm{CD} 45^{+} \mathrm{CD} 11 \mathrm{c}^{+}\right)$, putative MDSCs $\left(\mathrm{CD} 45^{+} \mathrm{CD} 11 \mathrm{~b}^{+} \mathrm{Gr}-1^{+}\right)$, and macrophages $\left(\mathrm{CD} 45^{+} \mathrm{CD} 11 \mathrm{~b}^{+} \mathrm{Gr}-1^{-}\right)$. On the right panels, the tree is colored by the median intensity of individual markers shown on the top to highlight infiltrating immune cells $\left(\mathrm{EpCAM}^{-} \mathrm{CD}^{+} 5^{+}\right)$, epithelial PCa cells $\left(\mathrm{EpCAM}^{+} \mathrm{CD} 45^{-}\right)$, total myeloid cells $\left(\mathrm{CD} 45^{+}\right.$ $\left.\mathrm{CD} 11 \mathrm{~b}^{+}\right)$, and putative MDSCs $\left(\mathrm{CD} 45^{+} \mathrm{CD} 11 \mathrm{~b}^{+} \mathrm{Gr}-1^{+}\right)$. (B-C) CyTOF analysis of tumors

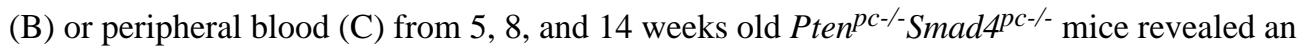
age-dependent increase in the MDSCs infiltration. Prostate from wild type (WT) mice at 16week old was used as control ( $n=3$ for each genotype). See also Supplementary Figure S1. 
A

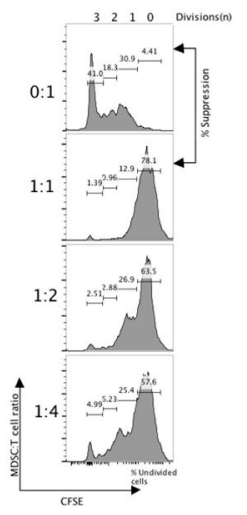

D

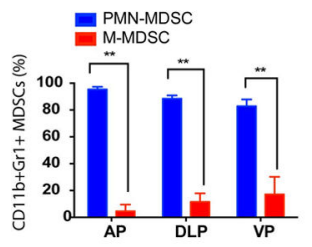

$\mathbf{F}$

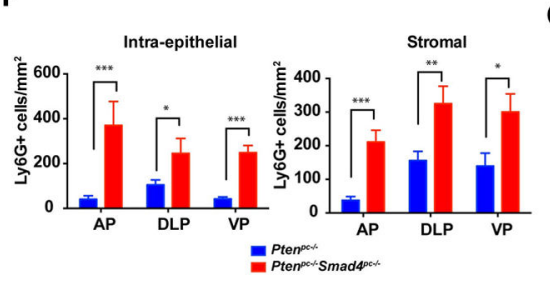

B
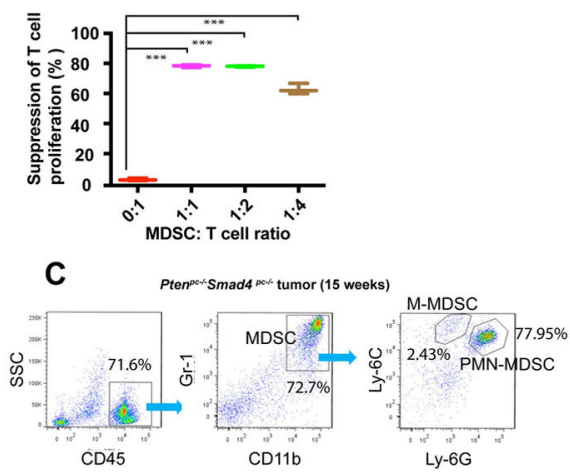

E

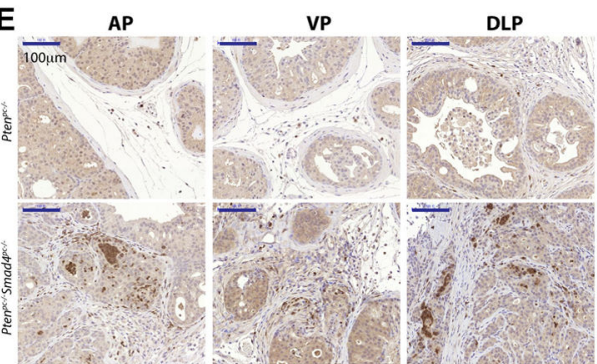

G

Figure 3. MDSCs from Pten ${ }^{p c-/-S m a d 4}{ }^{p c-/-}$ Tumors Display Potent Immunosuppressive Activities and are dominated by PMN-MDSCs

(A) $\mathrm{CD}_{11 \mathrm{~b}^{+}}$Gr- $1^{+}$cells from Pten ${ }^{\text {pc-/-Smad }} 4^{\text {pc-/- }}$ tumors display potent immune-suppressive activity towards $\mathrm{T}$ cell activation as demonstrated by CFSE dilution assay in triplicate. (B) Summarized result from (A). (C-D) Flow cytometry analysis shows PMN-MDSCs as the major population in the infiltrated MDSCs in established Pten $^{\text {pc-/-Smad4 }}{ }^{\text {pc-/- }}$ tumors at AP,

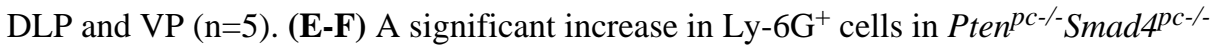
tumors as compared to the Pten ${ }^{p c-/-}$ tumors as shown by IHC for Ly-6G and quantified by location of positively stained cells in the intra-epithelial or stromal compartment of the tumor at AP, DLP and VP $(n=3)$. (G) Quantification of the mRNA expression of subunits of

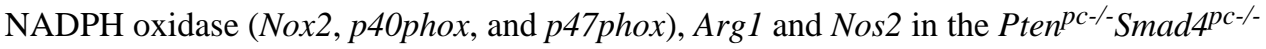
tumors and the Pten ${ }^{p c-/-}$ tumors $(\mathrm{n}=5)$. In $\mathrm{B}, \mathrm{D}, \mathrm{F}$ and $\mathrm{G}, * P<0.05$, $* * P<0.01$, $* * * P<0.001$. Also see Supplementary Figure S2. 


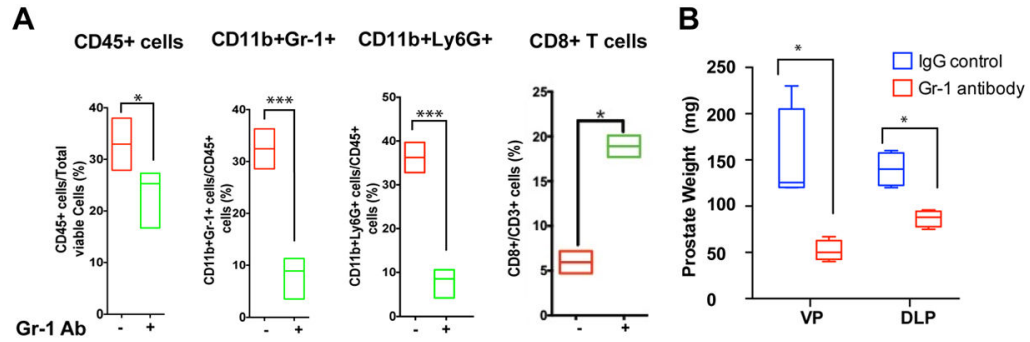

C

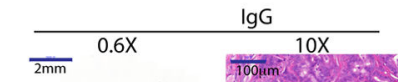

$\stackrel{2}{4}$

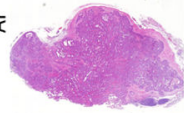

$s$

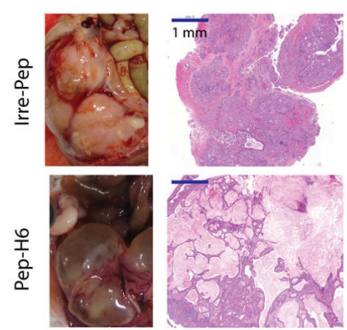

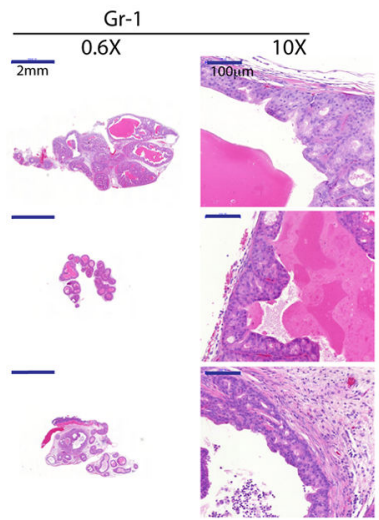

E

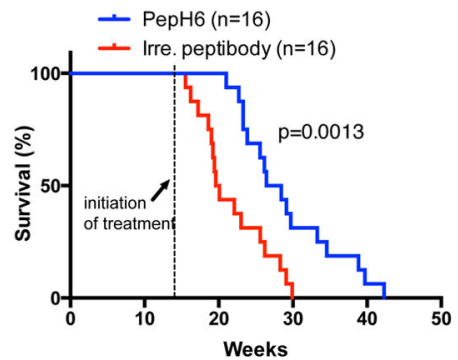

Figure 4. Targeting MDSCs with anti-Gr-1 Neutralizing Antibody or MDSC-specific Peptibody

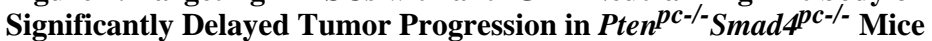

(A) Administration of Gr-1 neutralizing antibody in vivo significantly reduced CD45 ${ }^{+}$ infiltrating immune cells, reduced MDSCs and increased $\mathrm{CD}^{+} \mathrm{T}$ cells among total $\mathrm{T}$ cells in Pten ${ }^{\text {pc-/-Smad4 }}{ }^{\text {pc-/- }}$ tumors ( $\mathrm{n}=4$ ), measured by flow cytometry. (B) Gr-1 antibody treatment of 14-week old mice significantly reduced the weight of VP and DLP in Pten $^{\text {pc-- }}{ }^{-S m a d} 4^{\text {pc-l- }^{-}}$mice. (C) Gr-1 antibody remarkably altered the tumor histopathology in

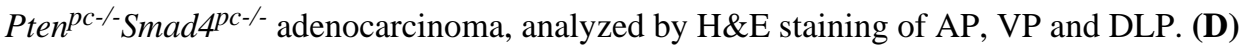
One month of Pep-H6 peptibody treatment led to significant appearance and histology changes of the Pten ${ }^{p c-/-S m a d 4}{ }^{p c-/}$ adenocarcinoma. (E) Kaplan-Meier survival curve showing the significant delay of mortality caused by Pep-H6 peptibody treatment of Pten ${ }^{p c-/}$ Smad 4 pc-/ mice. In A and B, $* P<0.05$, $* * * P<0.001$. Also see Supplementary Figure S3 and Figure S4. 
A

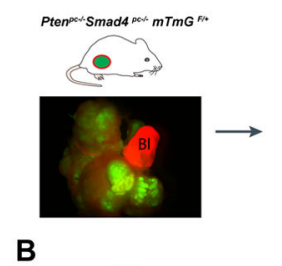

B
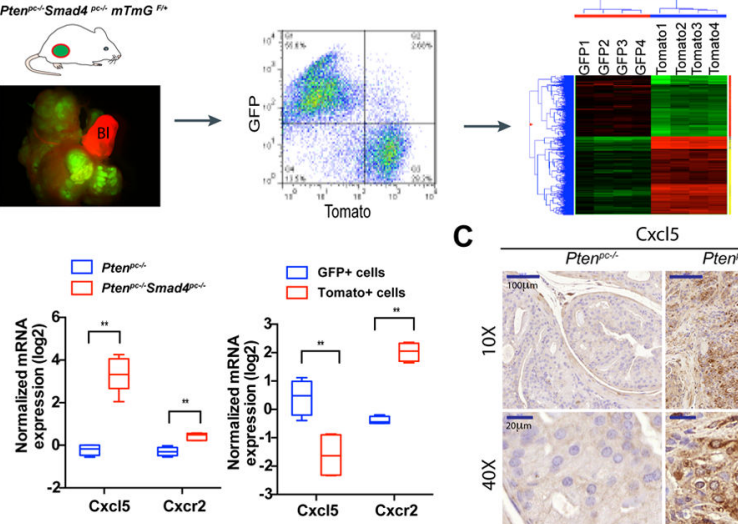

C
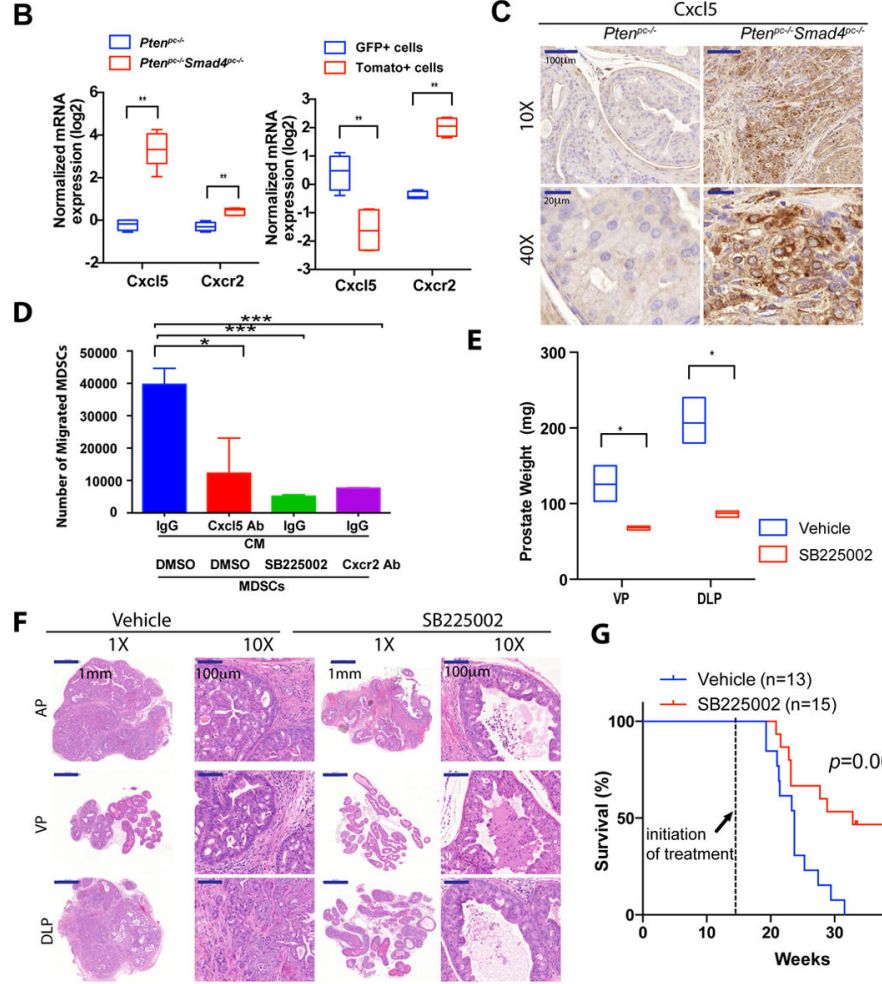

G

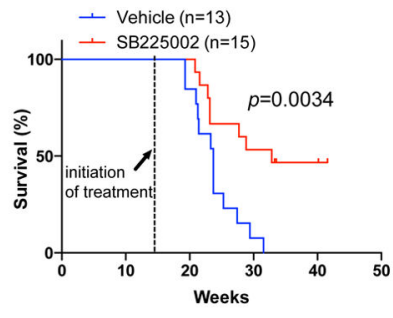

Figure 5. Cxcl5-Cxcr2 Axis Plays an Indispensable Role in Recruitment of MDSCs and Promotion of Tumor Progression

(A) Establishment of Pten ${ }^{p c-/-}$ Smad $4^{p c-/-} m T m G^{+}$model allows fluorescent visualization of the $\mathrm{GFP}^{+}$tumor cells intermixed with Tomato ${ }^{+}$stroma (Left Panel); FACS isolation of $\mathrm{GFP}^{+}$tumor cells and Tomato ${ }^{+}$stromal cells from the prostate adenocarcinoma (Middle Panel); microarray analysis to identify differentially expressed genes (Right Panel). In the fluorescence image, Bl denotes bladder (completely Tomato $\left.{ }^{+}\right)(n=2)$. (B) Quantification of mRNA expression shows that $\mathrm{Cxcl5}$ and $\mathrm{Cxcr} 2$ were both expressed at higher levels in

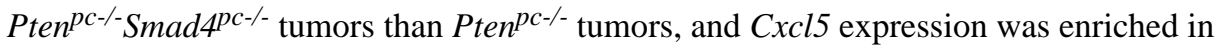
$\mathrm{GFP}^{+}$tumor cells, whereas $\mathrm{Cxcr} 2$ in Tomato ${ }^{+}$stromal cells. $(\mathrm{n}=5)(\mathbf{C}) \mathrm{IHC}$ for $\mathrm{Cxcl5}$ showed significantly higher expression levels of $\mathrm{Cxcl} 5$ in $\mathrm{Pten}^{\text {pc-/-Smad4pc-/- }}$ tumors than Pten $^{\text {pc-/- }}$ tumors $(\mathrm{n}=3)$. (D) Blocking the Cxcl5-Cxcr2 axis by $\mathrm{Cxcl5}$ neutralizing antibody, Cxcr2 inhibitor SB225002 or Cxcr2 neutralizing antibody significantly decreased migration of MDSCs towards conditioned medium from Pten ${ }^{p c-/-S m a d} 4^{p c-/-}$ tumors cells, evaluated with an in vitro transwell migration assay in triplicate. (E-F) Cxcr2 inhibitor SB225002 treatment of Pten ${ }^{p c-/-S m a d 4 p c-/-}$ mice for 14 days $(n=4)$ resulted in significantly reduced tumor weight of VP and DLP, and significantly delayed progression for AP prostate cancer

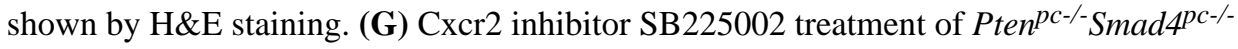


mice significantly prolonged their overall survival. In $\mathrm{B}, \mathrm{D}$ and $\mathrm{E}, * P<0.05, * * P<0.01$, $* * * P<0.001$.Also see Supplementary Figure S5. 
A
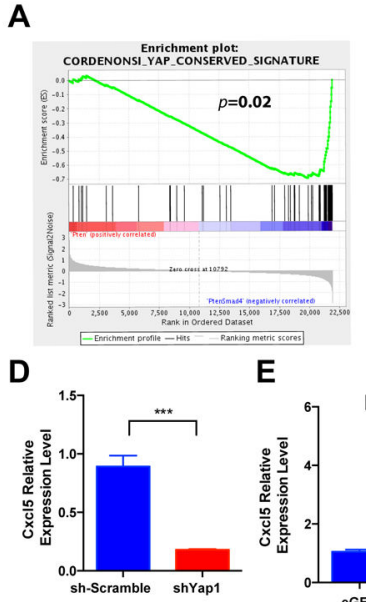

E
B
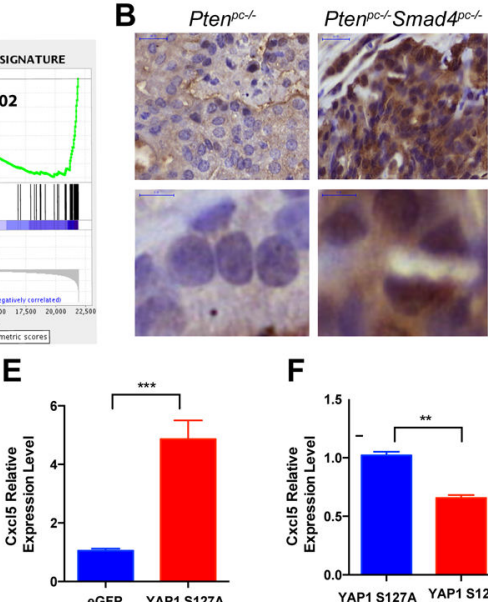

F

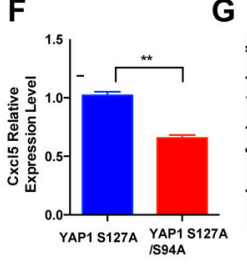

C
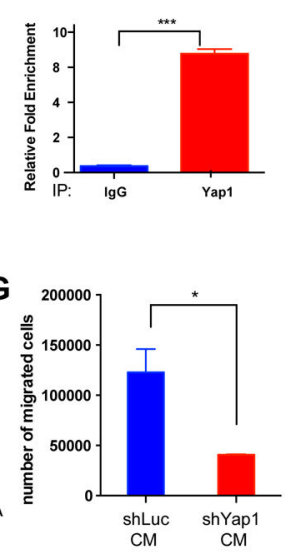

H

I

J
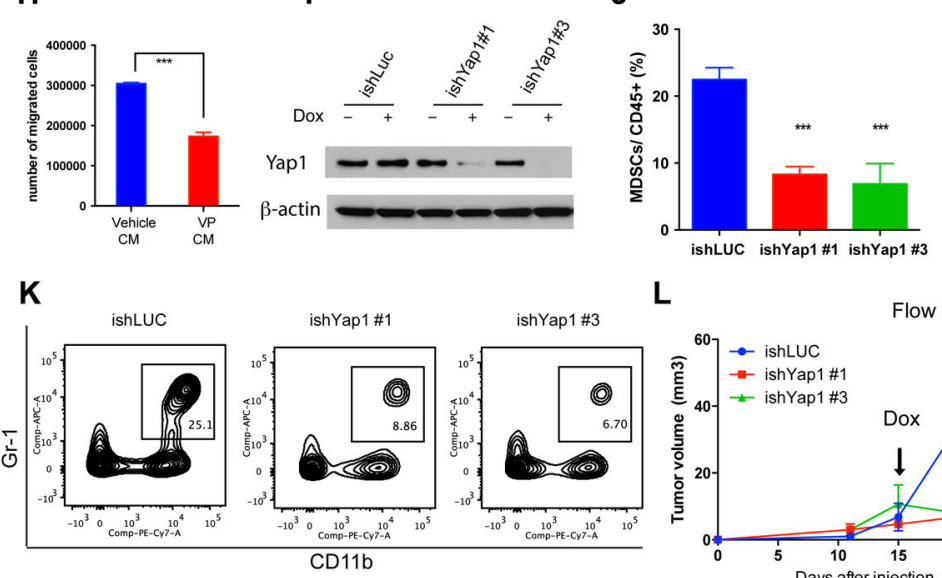

L

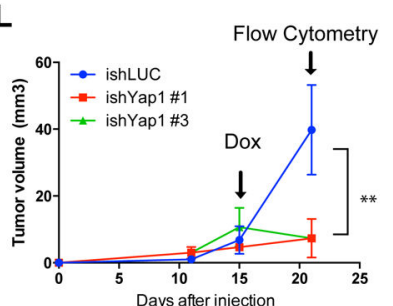

Figure 6. Hyperactivation of Yap1 in Pten ${ }^{\text {pc-/-S }}$ mad $^{\text {pc-/- }}$ Tumors Upregulates $\mathbf{C x c l 5}$

(A) GSEA analysis identified Yap1 oncogenic signature as the top activated pathways in the Pten $^{\text {pc-/-Smad }} 4^{\text {pc-/- }^{-}}$tumors compared to Pten ${ }^{\text {pc-/- }}$ tumors $(\mathrm{n}=5)$. (B) A significant increase in nuclear staining of Yap1 in the Pten ${ }^{\text {pc-/-Smad4 }}$ pc-/- $^{-}$tumors compared to Pten ${ }^{\text {pc-/- }}$ tumors $(\mathrm{n}=3)$. (C) Chromatin immunoprecipitation shows that Yap1 can directly bind to $\mathrm{Cxcl5}$ promoter using quantitative PCR in triplicates. (D) shRNA knockdown of Yap1 in Pten $^{\text {pc-/-Smad4pc-/- }}$ tumor cells resulted in a dramatic reduction in Cxcl5 mRNA expression using quantitative PCR in triplicates. (E) Overexpression of a constitutively active YAP1 S127A mutant resulted in upregulation of Cxcl5 mRNA using quantitative PCR in triplicates. (F) TEAD-binding defective S127A/S94A Yap1 mutant significantly decreased Cxcl5 mRNA expression as compared to the Yap1 S127A mutant using quantitative PCR in triplicates. (G-H) Conditioned medium prepared from Pten ${ }^{p c-/}$ Smad4 ${ }^{\text {pc-/- }}$ cells infected with Yap1 shRNA (G) or treated with Verteporfin $(\mathrm{H})$, a small molecule that disrupts Yap1TEAD interaction, induced less MDSCs migration in vitro as compared to the control conditioned medium. Transwell migration was done in triplicate for each condition. (I) Western blot analysis showed that two independent inducible shRNAs for Yap1 efficiently knockdown Yap1 expression in the Pten ${ }^{\text {pc-/-Smad4 }}{ }^{\text {pc-/- }}$ cells. (J-L) Inducible Yap1 knockdown strongly suppressed the intratumoral MDSC infiltration (J-K) and tumor growth 
(L) of the C57BL/6-syngeneic cell line isolated from prostate tumor of Pten $^{\text {pc-/-Smad4pc-/-Trp53 }}{ }^{\text {pc-/ }}$ mice (n=5). In $\mathbf{C}, \mathbf{D}, \mathbf{E}$ and $\mathbf{F}, \mathbf{G}, \mathbf{H}, \mathbf{L} * * P<0.01$, $* * * P<0.001$. See also Supplementary Figure S6. 


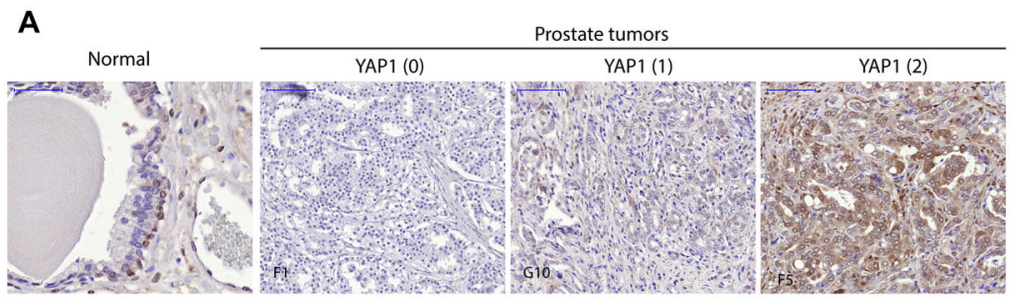

B
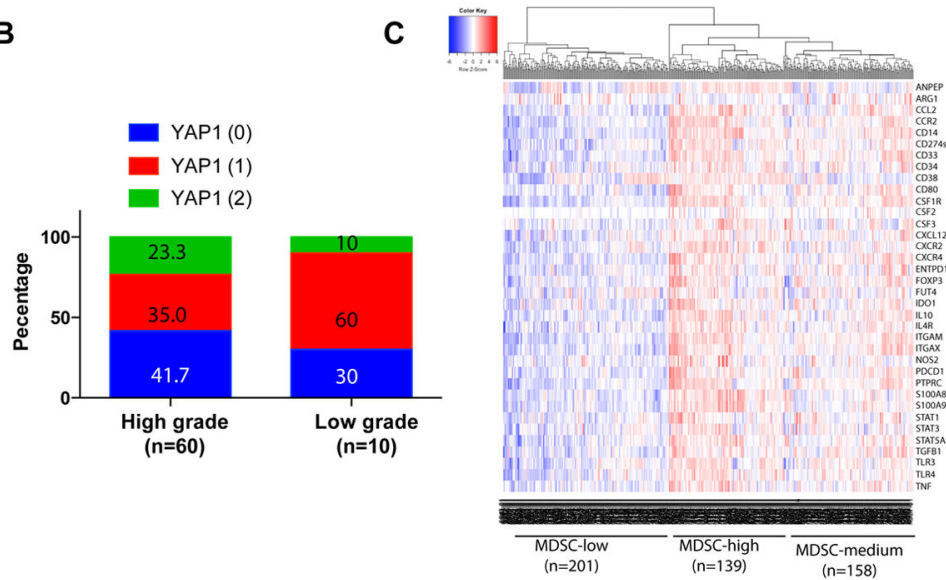

D

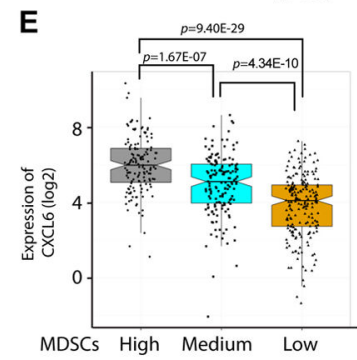

Figure 7. YAP1 is activated in human prostate cancer and correlated with MDSCs signature and CXCL6 overexpression

(A) IHC analysis of YAP1 expression in basal cells of normal prostate tissue and human prostate cancers. Numbers in parenthesis indicates YAP1 IHC intensity scores. (B) YAP1 IHC intensity score representation in low grade $(n=10)$ and high grade $(n=60)$ prostate cancer. (C) Clustering of human TCGA prostate samples into MDSC-high, MDSC-low and MDSC-medium groups using a 39-gene MDSC signature. (D) YAP1 signatures are identified in MDSC-high prostate TCGA samples. (E) CXCL6 expression is significantly higher in MDSC-high group. See also Supplementary Figure S7. 